ISSN 1855-3966 (printed edn.), ISSN 1855-3974 (electronic edn.)

ARS MATHEMATICA CONTEMPORANEA 16 (2019) 487-525

https://doi.org/10.26493/1855-3974.1330.916

(Also available at http://amc-journal.eu)

\title{
Linear separation of connected dominating sets in graphs*
}

\author{
Nina Chiarelli ${ }^{\dagger}$, Martin Milanič $\ddagger$ \\ University of Primorska, UP FAMNIT, Glagoljaška 8, SI-6000 Koper, Slovenia \\ University of Primorska, UP IAM, Muzejski trg 2, SI-6000 Koper, Slovenia
}

Received 21 February 2017, accepted 12 November 2018, published online 15 March 2019

\begin{abstract}
A connected dominating set in a graph is a dominating set of vertices that induces a connected subgraph. Following analogous studies in the literature related to independent sets, dominating sets, and total dominating sets, we study in this paper the class of graphs in which the connected dominating sets can be separated from the other vertex subsets by a linear weight function. More precisely, we say that a graph is connected-domishold if it admits non-negative real weights associated to its vertices such that a set of vertices is a connected dominating set if and only if the sum of the corresponding weights exceeds a certain threshold. We characterize the graphs in this non-hereditary class in terms of a property of the set of minimal cutsets of the graph. We give several characterizations for the hereditary case, that is, when each connected induced subgraph is required to be connected-domishold. The characterization by forbidden induced subgraphs implies that the class properly generalizes two well known classes of chordal graphs, the block graphs and the trivially perfect graphs. Finally, we study certain algorithmic aspects of connected-domishold graphs. Building on connections with minimal cutsets and properties of the derived hypergraphs and Boolean functions, we show that our approach leads to new polynomially solvable cases of the weighted connected dominating set problem.
\end{abstract}

Keywords: Connected dominating set, connected domination, connected-domishold graph, forbidden induced subgraph characterization, split graph, chordal graph, minimal cutset, minimal separator, 1-Sperner hypergraph, threshold hypergraph, threshold Boolean function, polynomial-time algorithm.

\footnotetext{
* The authors are grateful to the anonymous reviewers for the many insightful remarks, to Endre Boros, Andreas Brandstädt, Yves Crama, Vladimir Gurvich, Pinar Heggernes, Haiko Müller, and Vito Vitrih for helpful discussions, and to Dieter Kratsch and Haiko Müller for providing them with a copy of paper [43]. The work for this paper was partly done in the framework of a bilateral project between Argentina and Slovenia, financed by the Slovenian Research Agency (BI-AR/15-17-009) and MINCYT-MHEST (SLO/14/09).

Part of this work appeared as an extended abstract in [17].

${ }^{\dagger}$ This work is supported in part by the Slovenian Research Agency (research project J1-7051).

$\ddagger$ This work is supported in part by the Slovenian Research Agency (research core funding No. I0-0035 and P1-0285, and projects N1-0032, J1-7051, and J1-9110).
} 


\section{Introduction}

\subsection{Background}

Threshold concepts have been a subject of investigation for various discrete structures, including graphs [18,20,48], Boolean functions [19,22,29,32,53,55], and hypergraphs [34, 58]. A common theme of these studies is a quest for necessary and sufficient conditions for the property that a given combinatorial structure defined over some finite ground set $U$ admits non-negative real weights associated to elements of $U$ such that a subset of $U$ satisfies a certain property, say $\pi$, if and only if the sum of the corresponding weights exceeds a certain threshold. A more general framework has also been proposed, where the requirement is that a subset of $U$ satisfies property $\pi$ if and only if the sum of the corresponding weights belongs to a set $T$ of thresholds given by a membership oracle [50].

Having the set $U$ equipped with weights as above can have useful algorithmic implications. Consider for example the optimization problem of finding a subset of $U$ with property $\pi$ that has either maximum or minimum cost (according to a given linear cost function on the elements of the ground set). It was shown in [50] that if the weights as above are known and integer, then the problem can be solved by a dynamic programming approach in time $\mathcal{O}(|U| M)$ and with $M$ calls of the membership oracle, where $M$ is a given upper bound for $T$. The pseudo-polynomial running time should be expected, since the problem is very general and captures also the well-known knapsack problem [41]. Note, however, that the problem admits a much simpler, polynomial-time solution in the special case when the costs are unit and if we assume the monotone framework, where a set satisfies property $\pi$ as soon as its total weight exceeds a certain threshold. Under these assumptions, a minimum-sized subset of $U$ satisfying property $\pi$ can be found by a simple greedy algorithm starting with the empty set and adding the elements in order of non-increasing weight until the threshold is exceeded.

Many interesting graph classes can be defined within the above framework, including threshold graphs [20,42,48], domishold graphs [1], total domishold graphs [16, 18], equistable graphs [54], and equidominating graphs [54]. In general, the properties of the resulting graph classes depend both on the choice of property $\pi$ and on the constraints imposed on the structure of the set $T$ of thresholds. For example, if $U$ is the vertex set of a graph, property $\pi$ denotes the property of being an independent (stable) set in a graph, and $T$ is restricted to be an interval unbounded from below, we obtain the class of threshold graphs, which is a very well understood class of graphs, admitting many characterizations and linear-time algorithms for recognition and various optimization problems (see, e.g., [48]). If $\pi$ denotes the property of being a dominating set and $T$ is an interval unbounded from above, we obtain the class of domishold graphs, which enjoys similar properties as the class of threshold graphs. On the other hand, if $\pi$ is the property of being a maximal stable set and $T$ is restricted to consist of a single number, we obtain the class of equistable graphs, for which the recognition complexity is open (see, e.g., [47]), no structural characterization is known, and several NP-hard optimization problems remain intractable [50].

Notions and results from the theory of Boolean functions [22] and hypergraphs [2] can

E-mail addresses: nina.chiarelli@famnit.upr.si (Nina Chiarelli), martin.milanic@upr.si (Martin Milanič) 
be useful for the study of graph classes defined within the above framework. For instance, the characterization of hereditarily total domishold graphs in terms of forbidden induced subgraphs given in [18] is based on the facts that every threshold Boolean function is 2-asummable [19] and that every dually Sperner hypergraph is threshold [16]. ${ }^{1}$ Moreover, the fact that threshold Boolean functions are closed under dualization and (when given by their complete DNF) can be recognized in polynomial time [55] leads to efficient algorithms for recognizing total domishold graphs and for finding a minimum total dominating set in a given total domishold graph [16]. The relationship also goes the other way around. For instance, total domishold graphs can be used to characterize threshold hypergraphs and threshold Boolean functions [18].

\subsection{Aims and motivation}

The aim of this paper is to further explore and exploit this fruitful interplay between threshold concepts in graphs, hypergraphs, and Boolean functions. We do this by studying the class of connected-domishold graphs, a new class of graphs that can be defined in the above framework, as follows. A connected dominating set (CD set for short) in a connected graph $G$ is a set $S$ of vertices of $G$ that is dominating, that is, every vertex of $G$ is either in $S$ or has a neighbor in $S$, and connected, that is, the subgraph of $G$ induced by $S$ is connected. The ground set $U$ is the vertex set of a connected graph $G=(V, E)$, property $\pi$ is the property of being a connected dominating set in $G$, and $T$ is any interval unbounded from above.

Our motivations for studying the notion of connected domination in the above threshold framework are twofold. First, connected domination is one of the most basic of the many variants of domination, with applications in modeling wireless networks, see, e.g., [6,11, $12,26,27,31,35,36,60-62,66]$. The connected dominating set problem is the problem of finding a minimum connected dominating set in a given connected graph. This problem is NP-hard (and hard to approximate) for general graphs and remains intractable even under significant restrictions, for instance, for the class of split graphs (see Section 6.2). On the other hand, as outlined above, the problem is polynomially solvable in the class of connected-domishold graphs equipped with weights as in the definition. This motivates the study of connected-domishold graphs. In particular, identification of subclasses of connected-domishold graphs might lead to new classes of graphs where the connected dominating set problem (or its weighted version) is polynomially solvable.

Second, despite the increasingly large variety of graph domination concepts studied in the literature (see, e.g., [35,36]), so far a relatively small number of "threshold-like" graph classes was studied with respect to notions of domination: the classes of domishold and equidominating graphs (corresponding to the usual domination), the class of equistable graphs (corresponding to independent domination), and the class of total domishold graphs (corresponding to total domination). These graph classes differ significantly with respect to their structural and algorithmic properties. For instance, while the class of domishold graphs is a highly structured hereditary subclass of cographs, the classes of equistable and of total domishold graphs are not contained in any nontrivial hereditary class of graphs and are not structurally understood..$^{2}$ In particular, the class of total domishold graphs is as rich in its combinatorial structure as the class of threshold hypergraphs [18], for which (despite

\footnotetext{
${ }^{1}$ In $[16,18]$, the hereditarily total domishold graphs were named hereditary total domishold graphs. We prefer to adopt the grammatically correct term "hereditarily total domishold".

${ }^{2} \mathrm{~A}$ class of graphs is said to be hereditary if it is closed under vertex deletion.
} 
being recognizable in polynomial time via linear programming $[22,55]$ ) the existence of a "purely combinatorial" polynomial-time recognition algorithm is an open problem [22]. These results, differences, and challenges provide further motivation for the study of structural and algorithmic properties of connected-domishold graphs.

\subsection{The definition}

Since a disconnected graph $G$ does not have any connected dominating sets, we restrict our attention to connected graphs in the following definition.

Definition 1.1. A connected graph $G=(V, E)$ is said to be connected-domishold ( $C D$ for short) if there exists a pair $(w, t)$ where $w: V \rightarrow \mathbb{R}_{+}$is a weight function and $t \in \mathbb{R}_{+}$is a threshold such that for every subset $S \subseteq V, w(S):=\sum_{x \in S} w(x) \geq t$ if and only if $S$ is a connected dominating set in $G$. Such a pair $(w, t)$ will be referred to as a connecteddomishold (CD) structure of $G$.

We emphasize that the class of connected-domishold graphs is not the intersection of the classes of connected and domishold graphs. In fact, the two classes are incomparable: the 4-vertex cycle is connected and domishold [1] but not connected-domishold, see Example 1.3 below; the 4 -vertex path is connected-domishold but not domishold. The hyphen in the name is meant to indicate this fact.

Example 1.2. The complete graph of order $n$ is connected-domishold. Indeed, any nonempty subset $S \subseteq V\left(K_{n}\right)$ is a connected dominating set of $K_{n}$, and the pair $(w, 1)$ where $w(x)=1$ for all $x \in V\left(K_{n}\right)$ is a CD structure of $K_{n}$.

Example 1.3. The 4-cycle $C_{4}$ is not connected-domishold. Denoting its vertices by $v_{1}, v_{2}$, $v_{3}, v_{4}$ in a cyclic order, we see that a subset $S \subseteq V\left(C_{4}\right)$ is $\mathrm{CD}$ if and only if it contains an edge. Therefore, if $(w, t)$ is a CD structure of $C_{4}$, then $w\left(v_{i}\right)+w\left(v_{i+1}\right) \geq t$ for all $i \in\{1,2,3,4\}$ (indices modulo 4), which implies $w\left(V\left(C_{4}\right)\right) \geq 2 t$. On the other hand, $w\left(v_{1}\right)+w\left(v_{3}\right)<t$ and $w\left(v_{2}\right)+w\left(v_{4}\right)<t$, implying $w\left(V\left(C_{4}\right)\right)<2 t$.

\subsection{Overview of results}

Our results can be divided into four interrelated parts and can be summarized as follows:

1) Characterizations in terms of derived hypergraphs (resp., derived Boolean functions); a necessary and a sufficient condition.

In a previous work [18, Proposition 4.1 and Theorem 4.5], total domishold graphs were characterized in terms of thresholdness of a derived hypergraph and a derived Boolean function. We give similar characterizations of connected-domishold graphs (Proposition 3.4). The characterizations lead to a necessary and a sufficient condition for a graph to be connected-domishold, respectively, expressed in terms of properties of the derived hypergraph (equivalently: of the derived Boolean function; Corollary 3.5).

2) The case of split graphs. A characterization of threshold hypergraphs.

While the classes of connected-domishold and total domishold graphs are in general incomparable, we show that they coincide within the class of connected split graphs (Theorem 4.3). Building on this equivalence, we characterize threshold hypergraphs in terms of the connected-domisholdness property of a derived split graph (Theorem 4.4). 
We also give examples of connected split graphs showing that neither of the two conditions for a graph to be connected-domishold mentioned above (one necessary and one sufficient) characterizes this property.

\section{3) The hereditary case.}

We observe that, contrary to the classes of threshold and domishold graphs, the class of connected-domishold graphs is not hereditary. This motivates the study of so-called hereditarily connected-domishold graphs, defined as graphs every connected induced subgraph of which is connected-domishold. As our main result (Theorem 5.4), we give several characterizations of the class of hereditarily connected-domishold graphs. The characterizations in terms of forbidden induced subgraphs implies that the class of hereditarily connected-domishold graphs is a subclass of the class of chordal graphs properly containing two well known classes of chordal graphs, the class of block graphs and the class of trivially perfect graphs.

\section{4) Algorithmic aspects via vertex separators.}

Finally, we build on all these results, together with some known results from the literature on connected dominating sets and minimal vertex separators in graphs, to study certain algorithmic aspects of the class of connected-domishold graphs and their hereditary variant. We identify a sufficient condition, capturing a large number of known graph classes, under which the CD property can be recognized efficiently (Theorem 6.1). We also show that the same condition, when applied to classes of connected-domishold graphs, results in classes of graphs for which the weighted connected dominating set problem (which is NP-hard even on split graphs) is polynomial-time solvable (Theorem 6.5). This includes the classes of hereditarily connected-domishold graphs and $F_{2}$-free split graphs (see Figure 1), leading to new polynomially solvable cases of the problem.

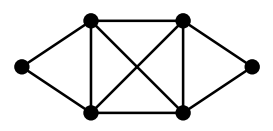

Figure 1: Graph $F_{2}$.

Structure of the paper. In Section 2, we state the necessary definitions and preliminary results on graphs, hypergraphs, and Boolean functions. In Section 3, we give characterizations of connected-domishold graphs in terms of thresholdness of derived hypergraphs and Boolean functions. Connected-domishold split graphs are studied in Section 4, where their relation to threshold hypergraphs is also observed. The main result of the paper, Theorem 5.4, is stated in Section 5, where some of its consequences are also derived. Section 6 discusses some algorithmic aspects of connected-domishold graphs. Our proof of Theorem 5.4 relies on a technical lemma, which is proved in Section 7.

\section{Preliminaries}

\subsection{Graphs}

All graphs in this paper will be finite, simple and undirected. The (open) neighborhood of a vertex $v$ is the set of vertices in a graph $G$ adjacent to $v$, denoted by $N_{G}(v)$ (or simply 
$N(v)$ if the graph is clear from the context); the closed neighborhood of $v$ is denoted by $N_{G}[v]$ and defined as $N_{G}(v) \cup\{v\}$. The degree of a vertex $v$ in a graph $G$ is the cardinality of its neighborhood. The complete graph, the path and the cycle of order $n$ are denoted by $K_{n}, P_{n}$ and $C_{n}$, respectively. A clique in a graph is a subset of pairwise adjacent vertices, and an independent (or stable) set is a subset of pairwise non-adjacent vertices. A universal vertex in a graph $G$ is a vertex adjacent to all other vertices. For a set $S$ of vertices in a graph $G$, we denote by $G[S]$ the subgraph of $G$ induced by $S$. For a set $\mathcal{F}$ of graphs, we say that a graph is $\mathcal{F}$-free if it does not contain any induced subgraph isomorphic to a member of $\mathcal{F}$. Given a graph $G$, a vertex $v \in V(G)$, and a set $U \subseteq V(G) \backslash\{v\}$, we say that $v$ dominates $U$ if $v$ is adjacent to every vertex in $U$.

The main notion that will provide the link between threshold Boolean functions and hypergraphs is that of cutsets in graphs. A cutset in a graph $G$ is a set $S \subseteq V(G)$ such that $G-S$ is disconnected. A cutset is minimal if it does not contain any other cutset. For a pair of disjoint vertex sets $A$ and $B$ in a graph $G$ such that no vertex in $A$ has a neighbor in $B$, an $A, B$-separator is a set of vertices $S \subseteq V(G) \backslash(A \cup B)$ such that $A$ and $B$ are in different components of $G-S$. An $A, B$-separator is said to be minimal if it does not contain any other $A, B$-separator. When sets $A$ and $B$ are singletons, say $A=\{u\}$ and $B=\{v\}$, we will refer to a (minimal) $A, B$-separator simply as a (minimal) $u, v$-separator. A minimal vertex separator in $G$ is a minimal $u, v$-separator for some non-adjacent vertex pair $u, v$. Note that every minimal cutset of $G$ is a minimal vertex separator, but not vice versa. The minimal cutsets are exactly the minimal $u, v$-separators that do not contain any other $x, y$-separator. The connection between the $\mathrm{CD}$ graphs and the derived hypergraphs and Boolean functions will be developed in Section 3 using the following characterization of CD sets due to Kanté et al. [38].

Proposition 2.1 (Kanté et al. [38]). In every connected graph $G$ that is not complete, a subset $D \subseteq V(G)$ is a $C D$ set if and only if $D \cap S \neq \emptyset$ for every minimal cutset $S$ in $G$.

In other words, unless a connected graph $G$ is complete, its CD sets are exactly the transversals of the cutset hypergraph of $G$ (see Section 2.3 and Definition 3.2 for definitions of these notions).

A graph $G$ is chordal if it does not contain any induced cycle of order at least 4, and split if it has a split partition, that is, a partition of its vertex set into a clique and an independent set. One of our proofs (the proof of Theorem 5.4) will rely on the following property of chordal graphs.

Lemma 2.2 (Kumar and Veni Madhavan [45]). If $S$ is a minimal cutset of a chordal graph $G$, then each connected component of $G-S$ has a vertex that is adjacent to all the vertices of $S$.

For graph theoretic notions not defined above, see, e.g., [65].

\subsection{Boolean functions}

Let $n$ be a positive integer. Given two vectors $x, y \in\{0,1\}^{n}$, we write $x \leq y$ if $x_{i} \leq y_{i}$ for all $i \in[n]:=\{1, \ldots, n\}$. A Boolean function $f:\{0,1\}^{n} \rightarrow\{0,1\}$ is positive (or: monotone) if $f(x) \leq f(y)$ holds for every two vectors $x, y \in\{0,1\}^{n}$ such that $x \leq y$. A literal of $f$ is either a variable, $x_{i}$, or the negation of a variable, denoted by $\overline{x_{i}}$. An elementary conjunction is an expression of the form $C=\left(\bigwedge_{i \in A} x_{i}\right) \wedge\left(\bigwedge_{j \in B} \overline{x_{j}}\right)$ where 
$A \cap B=\emptyset$. An implicant of a Boolean function $f$ is an elementary conjunction $C$ such that $f(x)=1$ for all $x \in\{0,1\}^{n}$ for which $C$ takes value 1 (we also say that $C$ implies $f$ ). An implicant is said to be prime if it is not implied by any other implicant. If $f$ is positive, then none of the variables appearing in any of its prime implicants appears negated. Every $n$-variable positive Boolean function $f$ can be expressed with its complete DNF (disjunctive normal form), defined as the disjunction of all prime implicants of $f$.

A positive Boolean function $f$ is said to be threshold if there exist non-negative real weights $w=\left(w_{1}, \ldots, w_{n}\right)$ and a non-negative real number $t$ such that for every $x \in\{0,1\}^{n}, f(x)=0$ if and only if $\sum_{i=1}^{n} w_{i} x_{i} \leq t$. Such a pair $(w, t)$ is called a separating structure of $f$. Every threshold Boolean function admits an integral separating structure (see [22, Theorem 9.5]). A positive Boolean function $f\left(x_{1}, \ldots, x_{n}\right)$ is threshold if and only if its dual function $f^{d}(x)=\overline{f(\bar{x})}$ is threshold [22]; moreover, if $\left(w_{1}, \ldots, w_{n}, t\right)$ is an integral separating structure of $f$, then $\left(w_{1}, \ldots, w_{n}, \sum_{i=1}^{n} w_{i}-t-1\right)$ is a separating structure of $f^{d}$.

Threshold Boolean functions have been characterized in [19] and [29], as follows. A false point of $f$ is an input vector $x \in\{0,1\}^{n}$ such that $f(x)=0$; a true point is defined analogously. For $k \geq 2$, a positive Boolean function $f:\{0,1\}^{n} \rightarrow\{0,1\}$ is said to be $k$-summable if, for some $r \in\{2, \ldots, k\}$, there exist $r$ (not necessarily distinct) false points of $f$, say, $x^{1}, x^{2}, \ldots, x^{r}$, and $r$ (not necessarily distinct) true points of $f$, say $y^{1}, y^{2}, \ldots, y^{r}$, such that $\sum_{i=1}^{r} x^{i}=\sum_{i=1}^{r} y^{i}$ (note that the sums are in $\mathbb{Z}^{n}$ and not in $\mathbb{Z}_{2}^{n}$, the $n$-dimensional vector space over $\operatorname{GF}(2))$. Function $f$ is said to be $k$-asummable if it is not $k$-summable, and it is asummable if it is $k$-asummable for all $k \geq 2$.

Theorem 2.3 (Chow [19], Elgot [29], see also [22, Theorem 9.14]). A positive Boolean function $f$ is threshold if and only if it is asummable.

The problem of determining whether a positive Boolean function given by its complete DNF is threshold is solvable in polynomial time, using dualization and linear programming (see [55] and [22, Theorem 9.16]). The algorithm tests if a polynomially sized derived linear program has a feasible solution, and in case of a yes instance, the solution found yields a separating structure of the given function. Using, e.g., Karmarkar's interior point method for linear programming [39], one can assure that a rational solution is found. This results in a rational separating structure, which can be easily turned into an integral one. We summarize this result as follows.

Theorem 2.4. There exists a polynomial-time algorithm for recognizing threshold Boolean functions given by the complete DNF. In case of a yes instance, the algorithm also computes an integral separating structure of the given function.

Remark 2.5. The existence of a "purely combinatorial" polynomial-time recognition algorithm for threshold Boolean functions (that is, one not relying on solving an auxiliary linear program) is an open problem [22].

A similar approach as the one outlined above shows that every connected-domishold graph has an integral CD structure; we will often use this fact in the paper. For further background on Boolean functions, we refer to the comprehensive monograph by Crama and Hammer [22]. 


\subsection{Hypergraphs}

A hypergraph is a pair $\mathcal{H}=(V, E)$ where $V$ is a finite set of vertices and $E$ is a set of subsets of $V$, called hyperedges [2]. When the vertex set or the hyperedge set of $\mathcal{H}$ will not be explicitly given, we will refer to them by $V(\mathcal{H})$ and $E(\mathcal{H})$, respectively. A transversal (or: hitting set) of $\mathcal{H}$ is a set $S \subseteq V$ such that $S \cap e \neq \emptyset$ for all $e \in E$. A hypergraph $\mathcal{H}=(V, E)$ is threshold if there exist a weight function $w: V \rightarrow \mathbb{R}_{+}$and a threshold $t \in \mathbb{R}_{+}$such that for all subsets $X \subseteq V$, it holds that $w(X) \leq t$ if and only if $X$ contains no hyperedge of $\mathcal{H}$ [34]. Such a pair $(w, t)$ is said to be a separating structure of $\mathcal{H}$.

To every hypergraph $\mathcal{H}=(V, E)$, we can naturally associate a positive Boolean function $f_{\mathcal{H}}:\{0,1\}^{V} \rightarrow\{0,1\}$, defined by the positive DNF expression

$$
f_{\mathcal{H}}(x)=\bigvee_{e \in E} \bigwedge_{u \in e} x_{u}
$$

for all $x \in\{0,1\}^{V}$. Conversely, to every positive Boolean function $f:\{0,1\}^{n} \rightarrow\{0,1\}$ given by a positive DNF $\phi=\bigvee_{j=1}^{m} \bigwedge_{i \in C_{j}} x_{i}$, we can associate a hypergraph $\mathcal{H}(\phi)=$ $(V, E)$ as follows: $V=[n]$ and $E=\left\{C_{1}, \ldots, C_{m}\right\}$. It follows directly from the definitions that the thresholdness of hypergraphs and of Boolean functions are related as follows.

Proposition 2.6. A hypergraph $\mathcal{H}=(V, E)$ is threshold if and only if the positive Boolean function $f_{\mathcal{H}}$ is threshold. A positive Boolean function given by a positive DNF $\phi=\bigvee_{j=1}^{m} \bigwedge_{i \in C_{j}} x_{i}$ is threshold if and only if the hypergraph $\mathcal{H}(\phi)$ is threshold.

Applying Theorem 2.3 to the language of hypergraphs gives the following characterization of threshold hypergraphs. For $k \geq 2$, a hypergraph $\mathcal{H}=(V, E)$ is said to be $k$-summable if, for some $r \in\{2, \ldots, k\}$, there exist $r$ (not necessarily distinct) subsets $A_{1}, \ldots, A_{r}$ of $V$ such that each $A_{i}$ contains a hyperedge of $\mathcal{H}$, and $r$ (not necessarily distinct) subsets $B_{1}, \ldots, B_{r}$ of $V$ such that each $B_{i}$ does not contain a hyperedge of $\mathcal{H}$ and such that for every vertex $v \in V$, we have:

$$
\left|\left\{i: v \in A_{i}\right\}\right|=\left|\left\{i: v \in B_{i}\right\}\right|
$$

We say that a hypergraph $\mathcal{H}$ is $k$-asummable if it is not $k$-summable and it is asummable if it is $k$-asummable for all $k \geq 2$.

Corollary 2.7. A hypergraph $\mathcal{H}$ is threshold if and only if it is asummable.

A hypergraph $\mathcal{H}=(V, E)$ is said to be Sperner (or: a clutter) if no hyperedge of $\mathcal{H}$ contains another hyperedge, that is, if for every two distinct hyperedges $e$ and $f$ of $\mathcal{H}$, it holds that $\min \{|e \backslash f|,|f \backslash e|\} \geq 1$. Chiarelli and Milanič defined in [16,18] the notion of dually Sperner hypergraphs as the hypergraphs such that the inequality $\min \{|e \backslash f|,|f \backslash e|\} \leq 1$ holds for every pair of distinct hyperedges $e$ and $f$ of $\mathcal{H}$. It was proved in $[16,18]$ that dually Sperner hypergraphs are threshold; they were applied in the characterizations of total domishold graphs and their hereditary variant. Boros et al. introduced in [8] the following restriction of dually Sperner hypergraphs.

Definition 2.8 (Boros et al. [8]). A hypergraph $\mathcal{H}=(V, E)$ is said to be 1-Sperner if for every two distinct hyperedges $e$ and $f$ of $\mathcal{H}$, it holds that $\min \{|e \backslash f|,|f \backslash e|\}=1$. 
Note that a hypergraph is 1-Sperner if and only if it is both Sperner and dually Sperner. In particular, for Sperner hypergraphs the notions of dually Sperner and 1-Sperner hypergraphs coincide. Since a hypergraph $\mathcal{H}$ is threshold if and only if the Sperner hypergraph obtained from $\mathcal{H}$ by keeping only its inclusion-wise minimal hyperedges is threshold, the fact that dually Sperner hypergraphs are threshold is equivalent to the following fact, proved constructively by Boros et al. in [8] using a composition result for 1-Sperner hypergraphs developed therein.

Theorem 2.9 (Chiarelli and Milanič [18], Boros et al. [8]). Every 1-Sperner hypergraph is threshold.

\section{Connected-domishold graphs via hypergraphs and Boolean func- tions}

In a previous work [18, Proposition 4.1 and Theorem 4.5], total domishold graphs were characterized in terms of thresholdness of a derived hypergraph and a derived Boolean function. In this section we give similar characterizations of connected-domishold graphs.

We first recall some relevant definitions and a result from [18]. A total dominating set in a graph $G$ is a set $S \subseteq V(G)$ such that every vertex of $G$ has a neighbor in $S$. Note that only graphs without isolated vertices have total dominating sets. A graph $G=(V, E)$ is said to be total domishold (TD for short) if there exists a pair $(w, t)$ where $w: V \rightarrow \mathbb{R}_{+}$ is a weight function and $t \in \mathbb{R}_{+}$is a threshold such that for every subset $S \subseteq V$, $w(S):=\sum_{x \in S} w(x) \geq t$ if and only if $S$ is a total dominating set in $G$. A pair $(w, t)$ as above will be referred to as atal domishold (TD) structure of $G$. The neighborhood hypergraph of a graph $G$ is the hypergraph denoted by $\mathcal{N}(G)$ and defined as follows: the vertex set of $\mathcal{N}(G)$ is $V(G)$ and the hyperedge set consists precisely of the minimal neighborhoods in $G$, that is, of the inclusion-wise minimal sets in the family of neighborhoods $\{N(v): v \in V(G)\} .{ }^{3}$ Note that a set $S \subseteq V(G)$ is a total dominating set in $G$ if and only if it is a transversal of $\mathcal{N}(G)$.

Proposition 3.1 (Chiarelli and Milanič [18]). For a graph $G=(V, E)$, the following are equivalent:

\section{G is total domishold.}

2. Its neighborhood hypergraph $\mathcal{N}(G)$ is threshold.

The constructions of the derived hypergraph and the derived Boolean function used in our characterizations of connected-domishold graphs in terms of their thresholdness are specified by Definitions 3.2 and 3.3.

Definition 3.2. Given a graph $G$, the cutset hypergraph of $G$ is the hypergraph $\mathcal{C}(G)$ with vertex set $V(G)$ whose hyperedges are precisely the minimal cutsets in $G$.

Given a finite non-empty set $V$, we denote by $\{0,1\}^{V}$ the set of all binary vectors with coordinates indexed by $V$. Given a graph $G=(V, E)$ and a binary vector $x \in\{0,1\}$, its support set is the set denoted by $S(x)$ and defined by $S(x)=\left\{v \in V: x_{v}=1\right\}$. In

\footnotetext{
${ }^{3}$ In [18], the neighborhood hypergraph of $G$ was named reduced neighborhood hypergraph (of $G$ ) and denoted by $\mathcal{R N}(G)$. We changed the terminology in analogy with the term "cutset hypergraph", which will be introduced shortly.
} 
the following definition, we associate a Boolean function to a given $n$-vertex graph $G$. In order to avoid fixing a bijection between its vertex set and the set $[n]$, we will consider the corresponding Boolean function as being defined on the set $\{0,1\}^{V}$, where $V=V(G)$. Accordingly, a separating structure of such a Boolean function can be seen as a pair $(w, t)$ where $w: V \rightarrow \mathbb{R}^{+}$and $t \in \mathbb{R}^{+}$such that for every $x \in\{0,1\}^{V}$, we have $f(x)=0$ if and only if $\sum_{v \in S(x)} w(v) \leq t$.

Definition 3.3. Given a graph $G=(V, E)$, its cutset function is the positive Boolean function $f_{G}^{c u t}:\{0,1\}^{V} \rightarrow\{0,1\}$ that takes value 1 precisely on vectors $x \in\{0,1\}^{V}$ whose support set contains some minimal cutset of $G$.

The announced characterizations of connected-domishold graphs in terms of their cutset hypergraphs and cutset functions are given in the following proposition. The proof is based on two ingredients: the characterization of the connected dominating sets of a given connected (non-complete) graph given by Proposition 2.1 and the fact that threshold Boolean functions are closed under dualization.

Proposition 3.4. For a connected graph $G=(V, E)$, the following are equivalent:

1. G is connected-domishold.

2. Its cutset hypergraph $\mathcal{C}(G)$ is threshold.

3. Its cutset function $f_{G}^{c u t}$ is threshold.

Moreover, if $G$ is not a complete graph and $(w, t)$ is an integral separating structure of $f_{G}^{c u t}$ or of $\mathcal{C}(G)$, then $(w, w(V)-t)$ is a $C D$ structure of $G$.

Proof. We consider two cases, depending on whether $G$ is a complete graph or not.

Case 1: $G$ is complete.

In this case all the three statements hold. Recall that every complete graph is CD (see Example 1.2). Since complete graphs have no cutsets, the set of hyperedges of the cutset hypergraph $\mathcal{C}(G)$ is empty. Hence the hypergraph $\mathcal{C}(G)$ is threshold. The absence of (minimal) cutsets also implies that the cutset function $f_{G}^{c u t}$ is constantly equal to 0 and hence threshold.

Case 2: $G$ is not complete.

First we will show the equivalence between statements 1 and 3 . Since a positive Boolean function $f$ is threshold if and only if its dual function $f^{d}(x)=\overline{f(\bar{x})}$ is threshold, it suffices to argue that $G$ is connected-domishold if and only if $\left(f_{G}^{c u t}\right)^{d}$ is threshold.

We claim that for every $x \in\{0,1\}^{V}$, we have $\left(f_{G}^{c u t}\right)^{d}(x)=1$ if and only if $S(x)$, the support set of $x$, is a connected dominating set of $G$. Let $x \in\{0,1\}^{V}$ and let $S$ be the support set of $x$. By definition, $\left(f_{G}^{c u t}\right)^{d}(x)=1$ if and only if $f_{G}^{c u t}(\bar{x})=0$, which is the case if and only if $V \backslash S$ does not contain any minimal cutset of $G$. This is in turn equivalent to the condition that $S$ is a transversal of the cutset hypergraph of $G$, and, by Proposition 2.1, to the condition that $S$ is a connected dominating set of $G$. Therefore, $\left(f_{G}^{c u t}\right)^{d}(x)=1$ if and only if $S$ is a connected dominating set of $G$, as claimed.

Now, if $G$ is connected-domishold, then it has an integral connected-domishold structure, say $(w, t)$, and $(w, t-1)$ is a separating structure of the dual function $\left(f_{G}^{c u t}\right)^{d}$, which 
implies that $\left(f_{G}^{c u t}\right)^{d}$ is threshold. Conversely, if the dual function is threshold, with an integral separating structure $(w, t)$, then $(w, t+1)$ is a connected-domishold structure of $G$. This establishes the equivalence between statements 1 and 3 .

Next, we show the equivalence between statements 2 and 3. Note that the complete DNF of $f_{G}^{c u t}$, the cutset function of $G$, is given by the expression $\bigvee_{S \in \mathcal{C}(G)} \bigwedge_{u \in S} x_{u}$. It now follows directly from the definitions of threshold Boolean functions and threshold hypergraphs that function $f_{G}^{c u t}(x)$ is threshold if and only if cutset hypergraph $\mathcal{C}(G)$ is threshold.

Finally, if $(w, t)$ is an integral separating structure of $f_{G}^{c u t}$, then $(w, w(V)-t-1)$ is a separating structure of $\left(f_{G}^{c u t}\right)^{d}$ and hence $(w, w(V)-t)$ is a connected-domishold structure of $G$.

Recall that every 1-Sperner hypergraph is threshold (Theorem 2.9) and every threshold hypergraph is asummable (Corollary 2.7). Thus, in particular, every threshold hypergraph is 2-asummable. Applying these relations to the specific case of the minimal cutset hypergraphs, Proposition 3.4 leads to the following.

Corollary 3.5. For every connected graph $G$, the following holds:

1. If the cutset hypergraph $\mathcal{C}(G)$ is 1-Sperner, then $G$ is connected-domishold.

2. If $G$ is connected-domishold, then its cutset hypergraph $\mathcal{C}(G)$ is 2-asummable.

We will show in Section 4.1 that neither of the two statements in Corollary 3.5 can be reversed. On the other hand, in Section 5 we will prove that all the three properties become equivalent in the hereditary setting.

\section{Connected-domishold split graphs}

The following examples show that for general connected graphs, the CD and TD properties are incomparable:

- The path $P_{6}$ is connected-domishold (it has a unique minimal connected dominating set, formed by its internal vertices) but it is not total domishold (see, e.g., [18]).

- The graph in Figure 2 is TD but not CD.

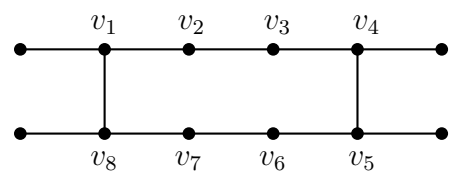

Figure 2: A TD graph that is not CD.

The graph is total domishold: it has a unique minimal total dominating set, namely $\left\{v_{1}, v_{4}, v_{5}, v_{8}\right\}$. On the other hand, the graph is not connected-domishold. This can be shown by observing that its cutset hypergraph is not 2-asummable and applying Corollary 3.5. To see that the cutset hypergraph of $G$ is 2 -summable, note that condition (2.1) is satisfied if we take $k=r=2$ and $A_{1}=\left\{v_{2}, v_{7}\right\}, A_{2}=\left\{v_{3}, v_{6}\right\}$, $B_{1}=\left\{v_{2}, v_{3}\right\}$, and $B_{2}=\left\{v_{6}, v_{7}\right\}$. 
Interestingly, we will show in Section 5 that if the CD and TD properties are required also for all connected induced subgraphs, then the corresponding graph classes become comparable (see Corollary 5.9). In the rest of this section, we will prove that the two properties coincide in the class of connected split graphs and examine some consequences of this result. Recall that a graph is split if and only if its vertex set has a partition into a clique and an independent set. Foldes and Hammer characterized split graphs as exactly the graphs that are $\left\{2 K_{2}, C_{4}, C_{5}\right\}$-free [30]. In particular, this implies that a split graph can be disconnected only if it has an isolated vertex.

Lemma 4.1. Let $G$ be a connected graph and let $G^{\prime}$ be the graph obtained from $G$ by adding to it a universal vertex. Then, $G$ is connected-domishold if and only if $G^{\prime}$ is connected-domishold.

Proof. Let $V\left(G^{\prime}\right)=V(G) \cup\{u\}$, where $u$ is the added vertex. Suppose that $G$ is connected-domishold and let $(w, t)$ be a CD structure of $G$. Since the set of connected dominating sets of $G^{\prime}$ consists of all connected dominating sets of $G$ together with all subsets of $V\left(G^{\prime}\right)$ containing $u$, we can obtain a CD structure, say $\left(w^{\prime}, t^{\prime}\right)$, of $G^{\prime}$ by setting $w^{\prime}(x)=w(x)$ for all $x \in V(G), w^{\prime}(u)=t$, and $t^{\prime}=t$. Therefore, $G^{\prime}$ is connecteddomishold.

Conversely, if $\left(w^{\prime}, t^{\prime}\right)$ is a CD structure of $G^{\prime}$, then $(w, t)$ where $t=t^{\prime}$ and $w$ is the restriction of $w^{\prime}$ to $V(G)$ is a CD structure of $G$. This is because a set $X \subseteq V(G)$ is a connected dominating set of $G$ if and only if it is a connected dominating set of $G^{\prime}$. Therefore, if $G^{\prime}$ is connected-domishold then so is $G$.

Recall that given a connected graph $G$, we denote by $\mathcal{C}(G)$ (resp., $\mathcal{N}(G)$ ) its cutset (resp., neighborhood) hypergraph.

Lemma 4.2. Let $G$ be a connected split graph without universal vertices. Then

$$
\mathcal{C}(G)=\mathcal{N}(G)
$$

Proof. Fix a split partition of $V(G)$, say $V(G)=K \cup I$ where $K$ is a clique, $I$ is an independent set, and $K \cap I=\emptyset$. Clearly, the hypergraphs $\mathcal{C}(G)$ and $\mathcal{N}(G)$ have the same vertex set. We show that the hyperedge sets are also the same in two steps.

First, we show that $E(\mathcal{C}(G)) \subseteq E(\mathcal{N}(G))$, that is, that every minimal cutset is a minimal neighborhood. To this end, it suffices to show that every minimal cutset $S$ in $G$ is a neighborhood, that is, a set of the form $S=N(v)$ for some $v \in V(G)$. This is indeed enough, because if a minimal cutset $S$ in $G$ satisfies $S=N(v)$ for some $v \in V(G)$, but $N(v)$ properly contains some other neighborhood, say $N(u)$, then the fact that $N(u)$ is a cutset in $G$ (for instance, it is a $u, v$-separator) would imply that $S$ is not a minimal cutset.

Let $S$ be a minimal cutset in $G$. Then, $S$ is a minimal $u, v$-separator for some nonadjacent vertex pair $u, v$; in particular, $S \subseteq V(G) \backslash\{u, v\}$. We claim that $N(u) \subseteq S$ or $N(v) \subseteq S$. Suppose that this is not the case. Then, there exist a neighbor of $u$, say $u^{\prime}$, such that $u^{\prime} \notin S$, and a neighbor of $v$, say $v^{\prime}$, such that $v^{\prime} \notin S$. Since $\left\{u, v, u^{\prime}, v^{\prime}\right\} \subseteq V(G) \backslash S$ and $u$ and $v$ are in different components of $G-S$, vertices $u^{\prime}$ and $v^{\prime}$ are distinct and nonadjacent. Thus, at least one of $u^{\prime}$ and $v^{\prime}$, say $u^{\prime}$, is in $I$. This implies that $u \in K$ and therefore $v \in I$, which implies that $v^{\prime} \in K$ and hence $\left(u, v^{\prime}, v\right)$ is a $u, v$-path in $G-S$, a contradiction. This shows that $N(u) \subseteq S$ or $N(v) \subseteq S$, as claimed. Since each of $N(u)$ and $N(v)$ is a $u, v$-separator, the fact that $S$ is a minimal $u, v$-separator implies that $S \in\{N(u), N(v)\}$. This completes the proof of the inclusion $E(\mathcal{C}(G)) \subseteq E(\mathcal{N}(G))$. 
It remains to show that $E(\mathcal{N}(G)) \subseteq E(\mathcal{C}(G))$. Let $S$ be a minimal neighborhood in $G$. Then $S=N(v)$ for some $v \in V(G)$. Since $v$ is not universal, the set $V(G) \backslash N[v]$ is non-empty. Therefore $S$ is a $v, w$-separator for any $w \in V(G) \backslash N[v]$; in particular, $S$ is a cutset in $G$. Suppose for a contradiction that $S$ is not a minimal cutset in $G$. Then $S$ properly contains some minimal cutset, say $S^{\prime}$, in $G$. By the first part of the proof, $S^{\prime}$ is of the form $S^{\prime}=N(z)$ for some $z \in V(G)$. However, since $N(z)$ is a neighborhood properly contained in $S=N(v)$, this contradicts the fact that $S$ is a minimal neighborhood.

Theorem 4.3. A connected split graph is connected-domishold if and only if it is total domishold.

Proof. If $G$ is complete, then $G$ is both connected-domishold and total domishold. So we may assume that $G$ is not complete. More generally, we show next that we may assume that $G$ does not have any universal vertices. Suppose that $G$ has a universal vertex, say $u$, and let $G^{\prime}=G-u$. By [18, Proposition 3.3], $G$ is TD if and only if $G^{\prime}$ is TD. If $G^{\prime}$ is not connected, then $\{u\}$ is the only minimal connected dominating set of $G$ and hence $G$ is connected-domishold in this case. Furthermore, $G$ is also total domishold: since $G^{\prime}$ is a disconnected $2 K_{2}$-free graph, $G^{\prime}$ has an isolated vertex. Therefore, by [18], $G^{\prime}$ is TD, and hence so is $G$. If $G^{\prime}$ is connected, then by Lemma 4.1, $G$ is CD if and only if $G^{\prime}$ is CD. Therefore, the problem of verifying whether the $\mathrm{CD}$ and the TD properties are equivalent for $G$ reduces to the same problem for $G^{\prime}$. An iterative application of the above argument eventually reduces the graph to either a graph where both properties hold or to a connected graph without universal vertices.

Now, let $G$ be a connected split graph without universal vertices. By Proposition 3.4, $G$ is connected-domishold if and only if its cutset hypergraph $\mathcal{C}(G)$ is threshold. By Proposition 3.1, $G$ is total domishold if and only if its neighborhood hypergraph $\mathcal{N}(G)$ is threshold. Therefore, to prove the theorem it suffices to show that $\mathcal{C}(G)=\mathcal{N}(G)$. But this was established in Lemma 4.2.

Theorem 4.3 implies another relation between connected-domishold (split) graphs and threshold hypergraphs, one that in a sense reverses the one stated in Proposition 3.4. Given a hypergraph $\mathcal{H}=(V, E)$, the split-incidence graph of $\mathcal{H}$ (see, e.g., [38]) is the split graph $G$ such that $V(G)=V \cup E, V$ is a clique, $E$ is an independent set, and $v \in V$ is adjacent to $e \in E$ if and only if $v \in e$.

Theorem 4.4. Let $\mathcal{H}=(V, E)$ be a hypergraph with $\emptyset \notin E$. Then $\mathcal{H}$ is threshold if and only if its split-incidence graph is connected-domishold.

Proof. Since $\emptyset \notin E$, the split-incidence graph of $\mathcal{H}$ is connected. It was shown in [18] that a hypergraph is threshold if and only if its split-incidence graph is total domishold. The statement of the theorem now follows from Theorem 4.3.

It might be worth pointing out that in view of Remark 2.5 and Theorem 4.4 , it is an open problem of whether there is a "purely combinatorial" polynomial-time algorithm for recognizing connected-domishold split graphs. Further issues regarding the recognition problem of CD graphs will be discussed in Section 6.1. 


\subsection{Examples related to Corollary 3.5}

We now show that neither of the two statements in Corollary 3.5 can be reversed. First we exhibit an infinite family of CD split graphs whose cutset hypergraphs are not 1-Sperner.

Example 4.5. Let $n \geq 4$ and let $G=K_{n}^{*}$ be the graph obtained from the complete graph $K_{n}$ by gluing a triangle on every edge. Formally,

$$
\begin{aligned}
& V(G)=\left\{u_{1}, \ldots, u_{n}\right\} \cup\left\{v_{i j}: 1 \leq i<j \leq n\right\} \text { and } \\
& E(G)=\left\{u_{i} u_{j}: 1 \leq i<j \leq n\right\} \cup\left\{u_{i} v_{j k}: 1 \leq j<k \leq n \text { and } i \in\{j, k\}\right\} .
\end{aligned}
$$

The graph $G$ is a $C D$ graph: setting

$$
w(x)= \begin{cases}1, & \text { if } x \in\left\{u_{1}, \ldots, u_{n}\right\} \\ 0, & \text { otherwise. }\end{cases}
$$

and $t=n-1$ results in a CD structure of $G$. On the other hand, the cutset hypergraph of $G$ is not 1 -Sperner. Since every pair of the form $\left\{u_{i}, u_{j}\right\}$ with $1 \leq i<j \leq n$ is a minimal cutset of $G$, the cutset hypergraph contains $\left\{u_{1}, u_{2}\right\}$ and $\left\{u_{3}, u_{4}\right\}$ as hyperedges and is therefore not 1-Sperner.

Next, we argue that there exists a split graph $G$ whose cutset hypergraph is 2-asummable but $G$ is not CD. As observed already in [18], the fact that not every 2-asummable positive Boolean function is threshold can be used to construct split graphs $G$ such that $\mathcal{N}(G)$ is 2 -asummable and $G$ is not total domishold. The existence of split graphs with claimed properties now follows from Theorem 4.3 and its proof. For the sake of self-containment, we describe an example of such a construction in Appendix A.

\section{The hereditary case}

In this section we present the main result of this paper, Theorem 5.4, which gives several characterizations of graphs all connected induced subgraphs of which are $\mathrm{CD}$, and derive some of its consequences. The proof of Theorem 5.4 relies on a technical lemma about chordal graphs, which will be proved in Section 7.

We start with an example showing that, contrary to the classes of threshold and domishold graphs, the class of connected-domishold graphs is not hereditary. We assume notation from Example 1.3.

Example 5.1. The graph $G$ obtained from $C_{4}$ by adding to it a new vertex, say $v_{5}$, and making it adjacent exactly to one vertex of the $C_{4}$, say to $v_{4}$, is CD: the (inclusion-wise) minimal CD sets of $G$ are $\left\{v_{1}, v_{4}\right\}$ and $\left\{v_{3}, v_{4}\right\}$, hence a CD structure of $G$ is given by $w\left(v_{2}\right)=w\left(v_{5}\right)=0, w\left(v_{1}\right)=w\left(v_{3}\right)=1, w\left(v_{4}\right)=2$, and $t=3$.

This motivates the following definition.

Definition 5.2. A graph $G$ is said to be hereditarily connected-domishold (hereditarily $C D$ for short) if every connected induced subgraph of $G$ is connected-domishold.

In general, for a property $\Pi$ of connected graphs, a graph is said to be hereditarily $\Pi$ if every connected induced subgraph of it satisfies $\Pi$. Characterizations of classes of hereditarily $\Pi$ graphs where $\Pi$ denotes the property that the graph has a connected dominating set 
inducing a graph with a certain property $\Pi^{\prime}$ were given, for various choices of property $\Pi^{\prime}$, by Michalak in [49]. In [57], Pržulj et al. gave characterizations of hereditarily ПI graphs where $\Pi$ denotes the property that the graph has a dominating pair of vertices (that is, a pair of vertices such that every path between them is dominating). The class of hereditarily connected-domishold graphs corresponds to the case when $\Pi$ is the property of being connected-domishold.

In order to state the technical lemma to be used in the proof of Theorem 5.4, we need some terminology. A diamond is a graph obtained from $K_{4}$ by deleting an edge. Given a diamond $D$, we will refer to its vertices of degree two as its tips and denote them as $t$ and $t^{\prime}$, and to its vertices of degree three as its centers and denote them as $c$ and $c^{\prime}$. The respective vertex sets will be denoted by $T$ and $C$. Similar notation will be used for diamonds denoted by $D_{1}$ or $D_{2}$.

Lemma 5.3 (Diamond Lemma). Let $G$ be a connected chordal graph. Suppose that $G$ contains two induced diamonds $D_{1}=\left(V_{1}, E_{1}\right)$ and $D_{2}=\left(V_{2}, E_{2}\right)$ such that:

(i) $C_{1} \cap C_{2}=\emptyset$.

(ii) If no vertex in $C_{1}$ is adjacent to a vertex in $C_{2}$, then there exists a $C_{1}, C_{2}$-separator in $G$ of size one.

(iii) For each $j \in\{1,2\}$ the tips (i.e., $t_{j}, t_{j}^{\prime}$ ) of $D_{j}$ belong to different components of $G-C_{j}$.

(iv) For $j \in\{1,2\}$ every component of $G-C_{j}$ has a vertex that dominates $C_{j}$.

Then $G$ has an induced subgraph isomorphic to $F_{1}, F_{2}$, or $H_{i}$ for some $i \geq 1$, where the graphs $F_{1}, F_{2}$, and a general member of the family $\left\{H_{i}\right\}$ are depicted in Figure 3.

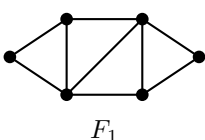

$F_{1}$

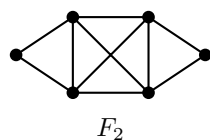

$F_{2}$

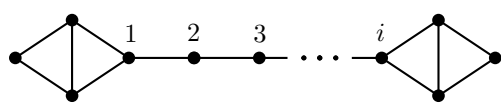

$H_{i} \quad(i \geq 1)$

Figure 3: Graphs $F_{1}, F_{2}$, and $H_{i}$.

The proof of Lemma 5.3 is postponed to Section 7.

Theorem 5.4. For every graph $G$, the following are equivalent:

1. G is hereditarily connected-domishold.

2. The cutset hypergraph of every connected induced subgraph of $G$ is 1-Sperner.

3. The cutset hypergraph of every connected induced subgraph of $G$ is threshold.

4. The cutset hypergraph of every connected induced subgraph of $G$ is 2-asummable.

5. $G$ is an $\left\{F_{1}, F_{2}, H_{1}, H_{2}, \ldots\right\}$-free chordal graph. 
Proof. The equivalence between items 1 and 3 follows from Proposition 3.4.

The implications $2 \Rightarrow 1 \Rightarrow 4$ follow from Corollary 3.5.

For the implication $4 \Rightarrow 5$, we only need to verify that the cutset hypergraph of none of the graphs in the set $\mathcal{F}:=\left\{C_{k}: k \geq 4\right\} \cup\left\{F_{1}, F_{2}\right\} \cup\left\{H_{i}: i \geq 1\right\}$ is 2-asummable. Let $F \in \mathcal{F}$. Suppose first that $F$ is a cycle $C_{k}$ for some $k \geq 4$, let $u_{1}, u_{2}, u_{3}, u_{4}$ be four consecutive vertices on the cycle. Let $A_{1}=\left\{u_{1}, u_{3}\right\}, A_{2}=\left\{u_{2}, u_{4}\right\}, B_{1}=\left\{u_{1}, u_{2}\right\}$ and $B_{2}=\left\{u_{3}, u_{4}\right\}$. Then, $A_{1}$ and $A_{2}$ are minimal cutsets of $F$ and thus hyperedges of the hypergraph $\mathcal{C}(F)$, while $B_{1}$ and $B_{2}$ do not contain any minimal cutset of $F$ and are consequently independent sets in the hypergraph $\mathcal{C}(F)$. Since the sets $A_{1}, A_{2}, B_{1}$ and $B_{2}$ satisfy condition (2.1), this implies that the hypergraph $\mathcal{C}(F)$ is 2 -summable. If $F \in$ $\left\{F_{1}, F_{2}\right\} \cup\left\{H_{i}: i \geq 1\right\}$, then let $a$ and $b$ be the two vertices of degree 2 in $F$, let $N(a)=\left\{a_{1}, a_{2}\right\}, N(b)=\left\{b_{1}, b_{2}\right\}$, let $A_{1}=N(a), A_{2}=N(b), B_{1}=\left\{a_{1}, b_{1}\right\}$ and $B_{2}=\left\{a_{2}, b_{2}\right\}$. The rest of the proof is the same as above.

It remains to show the implication $5 \Rightarrow 2$. Suppose that the implication fails and let $G$ be a minimal counterexample. That is, $G$ is an $\left\{F_{1}, F_{2}, H_{1}, H_{2}, \ldots\right\}$-free chordal graph such that its cutset hypergraph is not 1-Sperner, but the cutset hypergraph of every $\left\{F_{1}, F_{2}, H_{1}, H_{2}, \ldots\right\}$-free chordal graph with fewer vertices than $G$ is 1 -Sperner. Since $\mathcal{C}(G)$ is not 1 -Sperner, $G$ has two minimal cutsets, say $S$ and $S^{\prime}$, such that $\min \left\{\left|S \backslash S^{\prime}\right|,\left|S^{\prime} \backslash S\right|\right\} \geq 2$. The minimality of $G$ implies that the empty set is not a minimal cutset, hence $G$ is connected. Furthermore, the minimality ensures that $S$ and $S^{\prime}$ are disjoint sets (otherwise one can remove $S \cap S^{\prime}$ from $G$ and have a smaller counterexample). Thus, $\min \left\{|S|,\left|S^{\prime}\right|\right\} \geq 2$. The minimality also ensures that $|S|=\left|S^{\prime}\right|=2$. Indeed, removing a third vertex $z$, if present, from $S$ does not affect the minimal cutset status of $S$. Since every minimal cutset in a chordal graph is a clique [25], removing a third vertex $z$, if present, from $S$ will also not affect the minimal cutset status of $S^{\prime}$ since the entire $S$ (which is a clique) is present in one component of $G-S^{\prime}$.

The minimality also ensures that if there are no edges between $S$ and $S^{\prime}$, then every minimal $S, S^{\prime}$-separator $T$ is of size one. Indeed, if this is not the case, then $|T| \geq 2$ since $G$ is connected. Let $X$ be a component of $G-S$ containing $S^{\prime}$ and let $Y$ be any other component of $G-S$. The fact that $T$ separates $S$ from $S^{\prime}$ implies that $T$ contains all vertices in $N(S) \cap V(X)$, which is a non-empty set due to the minimality of $S$. Since $T$ is a minimal cutset in a chordal graph, it is a clique; in particular, it is fully contained in $X$. However, this implies that the sets $S^{\prime}$ and $T$ are minimal cutsets in the graph $G-V(Y)$ such that $\min \left\{\left|S^{\prime} \backslash T\right|,\left|T \backslash S^{\prime}\right|\right\} \geq 2$, contrary to the minimality of $G$.

Let $X, Y$ be two distinct components of $G-S$ and $X^{\prime}, Y^{\prime}$ two distinct components of $G-S^{\prime}$. By Lemma 2.2, there exist vertices $x \in X$ and $y \in Y$ such that each of $x$ and $y$ dominates $S$ and $x^{\prime} \in X^{\prime}$ and $y^{\prime} \in Y^{\prime}$ such that each of $x^{\prime}$ and $y^{\prime}$ dominates $S^{\prime}$. Let $D_{1}$ be the subgraph of $G$ induced by $S \cup\{x, y\}$ and let $D_{2}$ be the subgraph of $G$ induced by $S^{\prime} \cup\left\{x^{\prime}, y^{\prime}\right\}$. The definitions of $D_{1}$ and $D_{2}$ and Lemma 2.2 imply that $D_{1}$ and $D_{2}$ are two induced diamonds in $G$ satisfying the hypotheses of the Diamond Lemma (Lemma 5.3). Consequently, $G$ has an induced subgraph isomorphic to $F_{1}, F_{2}$, or $H_{i}$ for some $i \geq 1$, a contradiction. This completes the proof of the theorem.

Remark 5.5. The cutset hypergraph of a disconnected graph $H$ is equal to $(V(H),\{\emptyset\})$ and is clearly 1-Sperner (and therefore also threshold and 2-asummable). It follows that conditions from items $2-4$ in Theorem 5.4 are equivalent to the analogous conditions in which the respective properties are imposed on cutset hypergraphs of all induced subgraphs of $G$ (and not only of connected ones). 
In the rest of this section, we examine some of the consequences of the forbidden induced subgraph characterization of hereditarily CD graphs given by Theorem 5.4. The kite (also known as the co-fork or the co-chair) is the graph depicted in Figure 4.

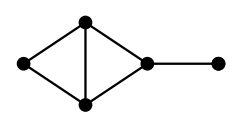

Figure 4: The kite.

The equivalence between items 1 and 5 in Theorem 5.4 implies that the class of hereditarily CD graphs is a proper generalization of the class of kite-free chordal graphs.

Corollary 5.6. Every kite-free chordal graph is hereditarily $C D$.

Corollary 5.6 further implies that the class of hereditarily CD graphs generalizes two well known classes of chordal graphs, the class of block graphs and the class of trivially perfect graphs. A graph is said to be a block graph if every block (maximal connected subgraph without cut vertices) of it is complete. The block graphs are well known to coincide with the diamond-free chordal graphs. A graph $G$ is said to be trivially perfect [33] if for every induced subgraph $H$ of $G$, it holds $\alpha(H)=|\mathcal{K}(H)|$, where $\alpha(H)$ denotes the independence number of $H$ (that is, the maximum size of an independent set in $H$ ) and $\mathcal{K}(H)$ denotes the set of all maximal cliques of $H$. Trivially perfect graphs coincide with the so-called quasi-threshold graphs [67], and are exactly the $\left\{P_{4}, C_{4}\right\}$-free graphs [33].

Corollary 5.7. Every block graph is hereditarily CD. Every trivially perfect graph is hereditarily $C D$.

Another class of graphs contained in the class of hereditarily CD graphs is the class of graphs defined similarly as the hereditarily CD graphs but with respect to total dominating sets. These so-called hereditarily total domishold graphs (abbreviated hereditarily TD graphs) were studied in [18], where characterizations analogous to those given by Theorem 5.4 were obtained, including the following characterization in terms of forbidden induced subgraphs.

Theorem 5.8 (Chiarelli and Milanič [18]). For every graph $G$, the following are equivalent:

1. G is hereditarily total domishold.

2. No induced subgraph of $G$ is isomorphic to a graph in Figure 5.

Theorems 5.4 and 5.8 imply the following.

Corollary 5.9. Every hereditarily $T D$ graph is hereditarily $C D$.

Proof. It suffices to verify that each of the forbidden induced subgraphs for the class of hereditarily connected-domishold graphs contains one of the graphs from Figure 5 as induced subgraph. A cycle $C_{k}$ with $k \geq 4$ contains (or is equal to) one of $C_{4}, C_{5}, C_{6}, P_{6}$. The graphs $F_{1}$ and $F_{2}$ are contained in both sets of forbidden induced subgraphs. Finally, each graph of the form $H_{i}$ where $i \geq 1$ contains $2 K_{3}$ as induced subgraph. 


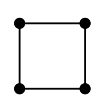

$C_{4}$

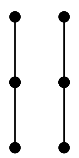

$F_{3}$

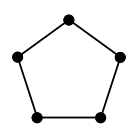

$C_{5}$

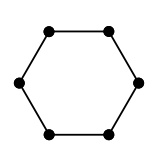

$C_{6}$

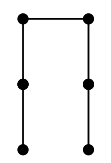

$P_{6}$

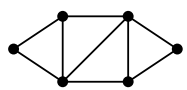

$F_{1}$

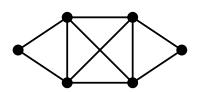

$F_{2}$

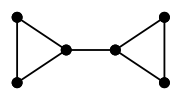

$F_{7}$

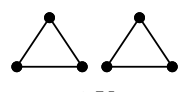

$2 K_{3}$

Figure 5: The set of forbidden induced subgraphs for the class of hereditarily total domishold graphs.

Since a graph is split if and only if it is $\left\{2 K_{2}, C_{4}, C_{5}\right\}$-free and each of the forbidden induced subgraphs for the class of hereditarily total domishold graphs other than $F_{2}$ contains either $2 K_{2}, C_{4}$, or $C_{5}$ as induced subgraph, Corollary 5.9 implies the following.

Corollary 5.10. Every $F_{2}$-free split graph is hereditarily $C D$.

Figure 6 shows a Hasse diagram depicting the inclusion relations among the class of hereditarily connected-domishold graphs and several other, well studied graph classes. All definitions of graph classes depicted in Figure 6 and the relations between them can be found in [23], with the exception of hereditarily CD and hereditarily TD graphs. The fact that every co-domishold graph is hereditarily TD and that every hereditarily TD graph is $(1,2)$-polar chordal was proved in [18]. The remaining inclusion and non-inclusion relations can be easily verified using the forbidden induced subgraph characterizations of the depicted graph classes, see [10,23,34].

\section{Algorithmic aspects via vertex separators}

In this section, we build on the above results, together with some known results from the literature on connected dominating sets and minimal vertex separators in graphs, to study certain algorithmic aspects of the class of connected-domishold graphs and its hereditary variant.

\subsection{The recognition problems}

We start with computational complexity aspects of the problems of recognizing whether a given graph is $\mathrm{CD}$, resp. hereditarily $\mathrm{CD}$. For general graphs, the computational complexity of recognizing connected-domishold graphs is not known. However, we will now show that the hypergraph approach outlined in Section 3 leads to a sufficient condition for the problem to be polynomially solvable, capturing a large number of graph classes. The condition is expressed using the notion of minimal vertex separators. Recall that a $u, v$-separator (for a pair of non-adjacent vertices $u, v)$ is a set $S \subseteq V(G) \backslash\{u, v\}$ such that $u$ and $v$ are in different components of $G-S$ and that a $u, v$-separator is minimal if it does not contain any other $u, v$-separator. Recall also that a minimal vertex separator in $G$ is a minimal $u, v$-separator for some non-adjacent vertex pair $u, v$. 


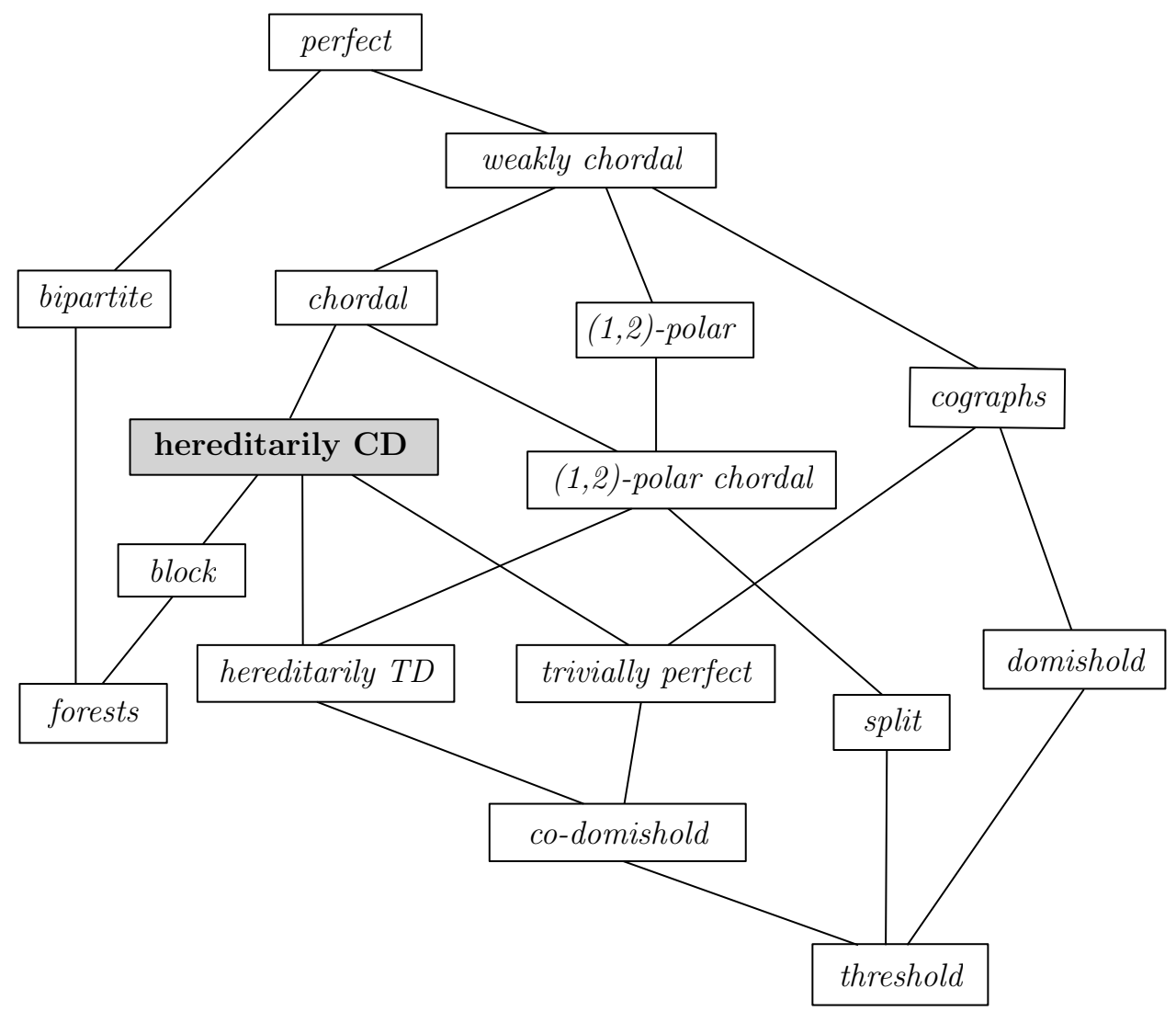

Figure 6: A Hasse diagram depicting the inclusion relations within several families of perfect graphs, focused around the class of hereditarily connected-domishold graphs. 
A sufficient condition for the polynomial-time solvability of the recognition problem for $\mathrm{CD}$ graphs in a class of graphs $\mathcal{G}$ is that there exists a polynomial $p$ such that every connected graph $G \in \mathcal{G}$ has at most $p(|V(G)|)$ minimal vertex separators. This is the case for chordal graphs, which have at most $|V(G)|$ minimal vertex separators [59], as well as for many other classes of graphs, including permutation graphs, circle graphs, circulararc graphs, chordal bipartite graphs, trapezoid graphs, cocomparability graphs of bounded dimension, distance-hereditary graphs, and weakly chordal graphs (see, e.g., [9, 43, 51]). For a polynomial $p$, let $\mathcal{G}_{p}$ be the class of graphs with at most $p(|V(G)|)$ minimal vertex separators. Since every minimal cutset is a minimal vertex separator, every connected graph $G \in \mathcal{G}_{p}$ has at most $p(|V(G)|)$ minimal cutsets.

It is known that the set of all minimal vertex separators of a given connected $n$-vertex graph can be enumerated in output-polynomial time. More precisely, Berry et al. [3] have developed an algorithm solving this problem in time $\mathcal{O}\left(n^{3}|\Sigma|\right)$ where $\Sigma$ is the set of all minimal vertex separators of $G$, improving on earlier (independently achieved) running times of $\mathcal{O}\left(n^{5}|\Sigma|\right)$ due to Shen and Liang [63] and Kloks and Kratsch [44]. Based on these results, we derive the following.

Theorem 6.1. For every polynomial $p$ there is a polynomial-time algorithm to determine whether a given connected graph $G \in \mathcal{G}_{p}$ is connected-domishold. In case of a yes instance, the algorithm also computes an integral CD structure of $G$.

Proof. Let $G=(V, E) \in \mathcal{G}_{p}$ be a connected graph that is the input to the algorithm.

The algorithm proceeds as follows. If $G$ is complete, then $G$ is connected-domishold and an integral CD structure of $G$ is returned, say $(w, t)$ with $w(x)=1$ for all $x \in V(G)$ and $t=1$. Assume now that $G$ is not complete. First, using the algorithm of Berry et al. [3], we compute in time $\mathcal{O}\left(|V(G)|^{3} p(|V(G)|)\right)$ the set $\Sigma$ of all minimal vertex separators of $G$. Next, the cutset hypergraph, $\mathcal{C}(G)$, is computed by comparing each pair of sets in $\Sigma$ and discarding the non-minimal ones. Since $\mathcal{C}(G)$ is Sperner, there is a bijective correspondence between the hyperedges of $\mathcal{C}(G)$ and the prime implicants of the cutset function $f_{G}^{c u t}$; this yields the complete DNF of $f_{G}^{c u t}$. Finally, we run the algorithm given by Theorem 2.4 on the complete DNF of $f_{G}^{c u t}$. If $f_{G}^{c u t}$ is not threshold, then we conclude that $G$ is not connected-domishold. Otherwise, the algorithm returned an integral separating structure, say $(w, t)$, of $f_{G}^{c u t}$. In this case we return $(w, w(V)-t)$ as a CD structure of $G$.

It is clear that the algorithm runs in polynomial time. Its correctness follows from Proposition 3.4.

Let $\tilde{\mathcal{G}}$ be the largest hereditary graph class such that a connected graph $G \in \tilde{\mathcal{G}}$ is connected-domishold if and only if it is total domishold. By Theorem 4.3, class $\tilde{\mathcal{G}}$ is a generalization of the class of split graphs. Since there is a polynomial-time algorithm for recognizing total domishold graphs [16, 18], there is a polynomial-time algorithm to determine whether a given connected graph $G \in \tilde{\mathcal{G}}$ is connected-domishold. This motivates the following question (which we leave open).

Question. What is the largest hereditary graph class $\tilde{\mathcal{G}}$ such that a connected graph $G \in \tilde{\mathcal{G}}$ is connected-domishold if and only if it is total domishold?

A polynomial-time recognition algorithm for the class of hereditarily CD graphs can be derived from the characterization of hereditarily $\mathrm{CD}$ graphs in terms of forbidden induced subgraphs given by Theorem 5.4. 
Proposition 6.2. There exists a polynomial-time algorithm to determine whether a given graph $G$ is hereditarily $C D$. In the case of a yes instance, an integral $C D$ structure of $G$ can be computed in polynomial time.

Proof. One can verify in linear time that $G$ is chordal [34] and verifying that $G$ is also $\left\{F_{1}, F_{2}, H_{1}, H_{2}\right\}$-free can be done in time $\mathcal{O}\left(|V(G)|^{8}\right)$. Therefore, we only have to show that we can check in polynomial time that $G$ does not contain an induced subgraph of the form $H_{i}$ for each $i>2$. Observe that for all $i>2$ the graph $H_{i}$ contains an induced subgraph isomorphic to $2 D$, the union of two diamonds (see Figure 3 and Figure 4). In $\mathcal{O}\left(\left|V(G)^{8}\right|\right)$ time, we can enumerate all induced subgraphs $F$ of $G$ isomorphic to $2 D$. For each such subgraph $F$ we have to verify whether it can be extended to an induced subgraph of the form $H_{i}$, for some $i>2$. We do this as follows. Let $D_{1}$ and $D_{2}$ be the connected components (diamonds) of $F$. Furthermore, let $u_{1}, u_{2}$ be the two vertices of degree 2 in $D_{1}$ and similarly let $v_{1}, v_{2}$ be the two vertices of degree 2 in $D_{2}$. Now we can verify that $F$ is not contained in any induced subgraph of $G$ isomorphic to $H_{i}$ (for some $i>2$ ) by checking for each pair $u_{i}, v_{j}$, with $i, j \in\{1,2\}$, that $u_{i}$ and $v_{j}$ belong to different components of $G-\left(N_{G-u_{i}}\left[V\left(D_{1}\right) \backslash\left\{u_{i}\right\}\right] \cup N_{G-v_{j}}\left[V\left(D_{2}\right) \backslash\left\{v_{j}\right\}\right]\right)$. This can be done in polynomial time and consequently the recognition of hereditarily CD graphs is a polynomially solvable problem.

The second part of the theorem follows from Theorem 6.1, since every hereditarily CD graph is chordal and chordal graphs are a subclass of $\mathcal{G}_{p}$ for the polynomial $p(n)=n[59]$.

It might seem conceivable that a similar approach as the one used in Theorem 6.1 could be used to develop an efficient algorithm for recognizing connected-domishold graphs in classes of graphs with only polynomially many minimal connected dominating sets. However, it is not known whether there exists an output-polynomial-time algorithm for the problem of enumerating minimal connected dominating sets. In fact, as shown by Kanté et al. [38], even when restricted to split graphs, this problem is equivalent to the well-known TRANS-ENUM problem in hypergraphs, the problem of enumerating the inclusion-minimal transversals of a given hypergraph. The TRANS-ENUM problem has been intensively studied but it is still open whether there exists an output-polynomial-time algorithm for the problem (see, e.g., the survey [28]).

\subsection{The weighted connected dominating set problem}

The Weighted Connected Dominating SeT (WCDS) problem takes as input a connected graph $G$ together with a cost function $c: V(G) \rightarrow \mathbb{R}^{+}$, and the task is to compute a connected dominating set of minimum total cost, where the cost of a set $S \subseteq V(G)$ is defined, as usual, as $c(S)=\sum_{v \in S} c(v)$. The WCDS problem has been studied extensively due to its many applications in networking (see, e.g., [6,26,66]). The problem is NP-hard not only for general graphs [36] but also for split graphs [46], chordal bipartite graphs [52], circle graphs [40], and cocomparability graphs [14]. Polynomial-time algorithms for the problem were developed for interval graphs [15] and more generally for trapezoid graphs [64] and circular-arc graphs [15,37], as well as for distance-hereditary graphs [68].

In this section, we will identify further graph classes where the WCDS problem is polynomially solvable, including the class of $F_{2}$-free split graphs (see Figure 1). This result 
is interesting in view of the fact that for split graphs, the WCDS problem is not only NPhard but also hard to approximate, even in the unweighted case. This can be seen as follows: Let $\mathcal{H}=(V, E)$ be a Sperner hypergraph with $\emptyset, V \notin E$ and let $G$ be its split-incidence graph. Then $G$ is a connected split graph without universal vertices, hence $\mathcal{C}(G)=\mathcal{N}(G)$ by Lemma 4.2. It can be seen that the hyperedge set of $\mathcal{N}(G)$ is exactly $E$, and therefore Proposition 2.1 implies that the problem of finding a minimum connected dominating set in $G$ is equivalent to the HITTING SET problem in hypergraphs, the problem of finding a minimum transversal of a given hypergraph. This latter problem is known to be equivalent to the well-known SET COVER problem and hence inapproximable in polynomial time to within a factor of $(1-\epsilon) \log |V|$, for any $\epsilon>0$, unless $\mathrm{P}=\mathrm{NP}$ [24]. It follows that the WCDS problem is hard to approximate to within a factor of $(1-\epsilon) \log |V(G)|$ in the class of split graphs.

We will show that the WCDS problem is polynomially solvable in the class of hereditarily $\mathrm{CD}$ graphs; the result for $F_{2}$-free split graphs will then follow. Our approach is based on connections with vertex separators and Boolean functions. First, we recall the following known results about: (i) the relation between the numbers of prime implicants of a threshold Boolean function and its dual, and (ii) the complexity of dualizing threshold Boolean functions. These results were proved in the more general context of regular Boolean functions (as well as for other generalizations, see, e.g., [7]).

Theorem 6.3. Let $f$ be an $n$-variable threshold Boolean function having exactly $q$ prime implicants. Then:

1. (Bertolazzi and Sassano [5], Crama [21], see also [22, Theorem 8.29]) The dual function $f^{d}$ has at most $N$ prime implicants, where $N$ is the total number of variables in the complete DNF of $f$.

2. (Crama and Hammer [22, Theorem 8.28] and Peled and Simeone [56]) There is an algorithm running in time $\mathcal{O}\left(n^{2} q\right)$ that, given the complete DNF of $f$, computes the complete DNF of the dual function $f^{d}$.

The algorithm by Crama and Hammer [22] is already presented as having time complexity $\mathcal{O}\left(n^{2} q\right)$, while the one by Peled and Simeone [56] is claimed to run in time $\mathcal{O}(n q)$. However, since $f^{d}$ can have $\mathcal{O}(n q)$ prime implicants, the total size of the output is of the order $\mathcal{O}\left(n^{2} q\right)$. The time complexity $\mathcal{O}(n q)$ of the algorithm by Peled and Simeone relies on the assumption that the algorithm outputs the prime implicants of the dual function one by one, each time overwriting the previous prime implicant (with a constant number of operations per implicant on average).

The relation between the numbers of prime implicants of a threshold Boolean function and its dual given by Theorem 6.3 implies that classes of connected-domishold graphs with only polynomially many minimal cutsets are exactly the same as the classes of connecteddomishold graphs with only polynomially many minimal connected dominating sets. More precisely:

Lemma 6.4. Let $G=(V, E)$ be an n-vertex connected-domishold graph that is not complete. Let $\nu_{c}$ (resp. $\nu_{s}$ ) denote the number of minimal connected dominating sets (resp. of minimal cutsets) of $G$. Then $\nu_{s} \leq(n-2) \nu_{c}$ and $\nu_{c} \leq(n-2) \nu_{s}$.

Proof. By Proposition 3.4, the cutset function $f_{G}^{c u t}$ is threshold. Function $f_{G}^{c u t}$ is an $n$ variable function with exactly $\nu_{s}$ prime implicants in its complete DNF. Recall from the 
proof of Proposition 3.4 that the dual function $\left(f_{G}^{c u t}\right)^{d}$ takes value 1 precisely on the vectors $x \in\{0,1\}^{V}$ whose support is a connected dominating set of $G$. Therefore, the prime implicants of $\left(f_{G}^{c u t}\right)^{d}$ are in bijective correspondence with the minimal connected dominating sets of $G$ and the number of prime implicants of $\left(f_{G}^{c u t}\right)^{d}$ is exactly $\nu_{c}$. Since every minimal cutset of $G$ has at most $n-2$ vertices, Theorem 6.3 implies that $\nu_{c} \leq(n-2) \nu_{s}$, as claimed.

Conversely, since $f_{G}^{c u t}=\left(\left(f_{G}^{c u t}\right)^{d}\right)^{d}$, the inequality $\nu_{s} \leq(n-2) \nu_{c}$ can be proved by a similar approach, provided we show that every minimal connected dominating set of $G$ has at most $n-2$ vertices. But this is true since if $D$ is a connected dominating set of $G$ with at least $n-1$ vertices, with $V(G) \backslash\{u\} \subseteq D$ for some $u \in V(G)$, then a smaller connected dominating set $D^{\prime}$ of $G$ could be obtained by fixing an arbitrary spanning tree $T$ of $G[D]$ and deleting from $D$ an arbitrary leaf $v$ of $T$ such that $N_{G}(u) \neq\{v\}$. (Note that since $G$ is connected but not complete, it has at least three vertices, hence $T$ has at least two leaves.) This completes the proof.

We now have everything ready to derive the main result of this section. Recall that for a polynomial $p$, we denote by $\mathcal{G}_{p}$ the class of graphs with at most $p(|V(G)|)$ minimal vertex separators.

Theorem 6.5. For every nonzero polynomial p, the set of minimal connected dominating sets of an n-vertex connected-domishold graph from $\mathcal{G}_{p}$ has size at most $\mathcal{O}(n \cdot p(n))$ and can be computed in time $\mathcal{O}\left(n \cdot p(n) \cdot\left(n^{2}+p(n)\right)\right)$. In particular, the WCDS problem is solvable in polynomial time in the class of connected-domishold graphs from $\mathcal{G}_{p}$.

Proof. Let $p$ and $G$ be as in the statement of the theorem and let $\mathcal{C D}(G)$ be the set of minimal connected dominating sets of $G$. If $G$ is complete, then

$$
\mathcal{C D}(G)=\{\{v\}: v \in V(G)\}
$$

and thus $|\mathcal{C D}(G)|=n=\mathcal{O}(n \cdot p(n))$ (since the polynomial is nonzero). Otherwise, we can apply Lemma 6.4 to derive $|\mathcal{C D}(G)| \leq(n-2) \cdot p(n)$.

A polynomial-time algorithm to solve the WCDS problem for a given connecteddomishold graph $G \in \mathcal{G}_{p}$ with respect to a cost function $c: V(G) \rightarrow \mathbb{R}^{+}$can be obtained as follows. First, we may assume that $G$ is not complete, since otherwise we can return a set $\{v\}$ where $v$ is a vertex minimizing $c(v)$. We use a similar approach as in the proof of Theorem 6.1. Using the algorithm of Berry et al. [3], we compute in time $\mathcal{O}\left(n^{3} p(n)\right)$ the set $\Sigma$ of all minimal vertex separators of $G$. We can assume that each minimal vertex separator has its elements listed according to some fixed order of $V(G)$ (otherwise, we can sort them in time $\mathcal{O}(n \cdot p(n))$ using, e.g., bucket sort). The cutset hypergraph, $\mathcal{C}(G)$, is then computed by comparing each pair of sets in $\Sigma$ and discarding the non-minimal ones; this can be done in time $\mathcal{O}\left(n \cdot(p(n))^{2}\right)$. The cutset hypergraph directly corresponds to the complete DNF of the cutset function $f_{G}^{c u t}$.

The next step is to compute the complete DNF of the dual function $\left(f_{G}^{c u t}\right)^{d}$. By Theorem 6.3 , this can be done in time $\mathcal{O}\left(n^{2} \cdot p(n)\right)$. Since each term of the DNF is a prime implicant of $\left(f_{G}^{c u t}\right)^{d}$ and the prime implicants of $\left(f_{G}^{c u t}\right)^{d}$ are in bijective correspondence with the minimal connected dominating sets of $G$, we can read off from the DNF all the minimal connected dominating sets of $G$. The claimed time complexity follows.

Once the list of all minimal connected dominating sets is available, a polynomial-time algorithm for the WCDS problem on $(G, c)$ follows immediately. 
In the case of chordal graphs, we can improve the running time by using one of the known linear-time algorithms for listing the minimal vertex separators of a given chordal graph due to Kumar and Veni Madhavan [45], Chandran and Grandoni [13], and Berry and Pogorelcnik [4].

Theorem 6.6. Every $n$-vertex connected-domishold chordal graph has at most $\mathcal{O}\left(n^{2}\right)$ minimal connected dominating sets, which can be enumerated in time $\mathcal{O}\left(n^{3}\right)$. In particular, the WCDS problem is solvable in time $\mathcal{O}\left(n^{3}\right)$ in the class of connected-domishold chordal graphs.

Proof. Let $G$ be an $n$-vertex connected-domishold chordal graph. The theorem clearly holds for complete graphs, so we may assume that $G$ is not complete. Since $G$ is chordal, it has at most $n$ minimal vertex separators [59]; consequently, $G$ has at most $n$ minimal cutsets. Since $G$ is connected-domishold, it has at most $n(n-2)$ minimal connected dominating sets, by Lemma 6.4.

The minimal connected dominating sets of $G$ can be enumerated as follows. First, we compute all the $\mathcal{O}(n)$ minimal vertex separators of $G$ in time $\mathcal{O}(n+m)$ (where $m=|E(G)|)$ using one of the known algorithms for this problem on chordal graphs $[4,13$, 45]. Assuming again that each minimal vertex separator has its elements listed according to some fixed order of $V(G)$, we then eliminate those that are not minimal cutsets in time $\mathcal{O}\left(n^{3}\right)$, by directly comparing each of the $\mathcal{O}\left(n^{2}\right)$ pairs for inclusion.

The list of $\mathcal{O}(n)$ minimal cutsets of $G$ yields its cutset function, $f_{G}^{m s}$. The list of minimal connected dominating sets of $G$ can be obtained in time $\mathcal{O}\left(n^{3}\right)$ by dualizing $f_{G}^{m s}$ using one of the algorithms given by Theorem 6.3. The WCDS problem can now be solved in time $\mathcal{O}\left(n^{3}\right)$ by evaluating the cost of each of the $\mathcal{O}\left(n^{2}\right)$ minimal connected dominating sets and outputting one of minimum cost.

From Theorem 6.6 we derive two new polynomially solvable cases of the WCDS problem. Recall that the graphs $F_{1}, F_{2}$, and a general member of the family $\left\{H_{i}\right\}$ are depicted in Figure 3.

Corollary 6.7. The WCDS problem is solvable in time $\mathcal{O}\left(n^{3}\right)$ in the class of $\left\{F_{1}, F_{2}, H_{1}\right.$, $\left.\mathrm{H}_{2}, \ldots\right\}$-free chordal graphs and in particular in the class of $F_{2}$-free split graphs.

Proof. By Theorem 5.4, every $\left\{F_{1}, F_{2}, H_{1}, H_{2}, \ldots\right\}$-free chordal graphs is (hereditarily) CD so Theorem 6.6 applies. The statement for $F_{2}$-free split graphs follows from Corollary 5.10 .

We conclude this section with two remarks, one related to Theorem 6.6 and one related to Theorems 6.1 and 6.5.

Remark 6.8. The bound $\mathcal{O}\left(n^{2}\right)$ given by Theorem 6.6 on the number of minimal connected dominating sets in an $n$-vertex connected-domishold chordal graph is sharp. There exist $n$-vertex connected-domishold chordal graphs with $\Theta\left(n^{2}\right)$ minimal connected dominating sets. For instance, let $S_{n}$ be the split graph with $V\left(S_{n}\right)=K \cup I$ where $K=\left\{u_{1}, \ldots, u_{n}\right\}$ is a clique, $I=\left\{v_{1}, \ldots, v_{n}\right\}$ is an independent set, $K \cap I=\emptyset$, and for each $i \in[n]$, vertex $u_{i}$ is adjacent to all vertices of $I$ except $v_{i}$. Since every vertex in $I$ has a unique non-neighbor in $K$, we infer that $S_{n}$ is $F_{2}$-free. Therefore, by Corollary 5.10 graph $S_{n}$ is a (hereditarily) connected-domishold graph. Note that every set of the form $\left\{u_{i}, u_{j}\right\}$ where $1 \leq i<j \leq n$ is a minimal connected dominating set of $S_{n}$. It follows that $S_{n}$ has at least $\left(\begin{array}{l}n \\ 2\end{array}\right)=\Theta\left(\left|V\left(S_{n}\right)\right|^{2}\right)$ minimal connected dominating sets. 
Remark 6.9. Theorems 6.1 and 6.5 motivate the question of whether there is a polynomial $p$ such that every connected CD graph $G$ has at most $p(|V(G)|)$ minimal vertex separators. As shown by the following family of graphs, this is not the case. For $n \geq 2$, let $G_{n}$ be the graph obtained from the disjoint union of $n$ copies of the $P_{4}$, say $\left(x_{i}, a_{i}, b_{i}, y_{i}\right)$ for $i=1, \ldots, n$, by identifying all vertices $x_{i}$ into a single vertex $x$, all vertices $y_{i}$ into a single vertex $y$, and for each vertex $z$ other than $x$ or $y$, adding a new vertex $z^{\prime}$ and making it adjacent only to $z$. It is not difficult to see that $G_{n}$ has exactly two minimal CD sets, namely $\left\{a_{1}, \ldots, a_{n}\right\} \cup\left\{b_{1}, \ldots, b_{n}\right\} \cup\{v\}$ for $v \in\{x, y\}$. A CD structure of $G_{n}$ is given by $(w, t)$ where $t=4 n+1, w(x)=w(y)=1, w\left(a_{i}\right)=w\left(b_{i}\right)=2$ for all $i \in\{1, \ldots, n\}$ and $w(z)=0$ for all other vertices $z$. Therefore, $G_{n}$ is CD. However, $G_{n}$ has $4 n+2$ vertices and $2^{n}$ minimal $x, y$-separators, namely all sets of the form $\left\{c_{1}, \ldots, c_{n}\right\}$ where $c_{i} \in\left\{a_{i}, b_{i}\right\}$ for all $i$.

\section{Proof of Lemma 5.3 (Diamond Lemma)}

In the proof of the Diamond Lemma, we use the following notation. We write $u \sim v$ (resp. $u \nsim v$ ) to denote the fact that two vertices $u$ and $v$ are adjacent (resp. non-adjacent). Given two vertex sets $A$ and $B$ in a graph $G$, we denote by $e(A, B)$ the number of edges with one endpoint in $A$ and one endpoint in $B$. A pattern is a triple $(V, E, F)$ where $G=(V, E)$ is a graph and $F$ is a subset of non-adjacent vertex pairs of $G$. We say that a graph $G^{\prime}$ realizes a pattern $(V, E, F)$ if $V\left(G^{\prime}\right)=V$ and $E \subseteq E\left(G^{\prime}\right) \subseteq E \cup F$.

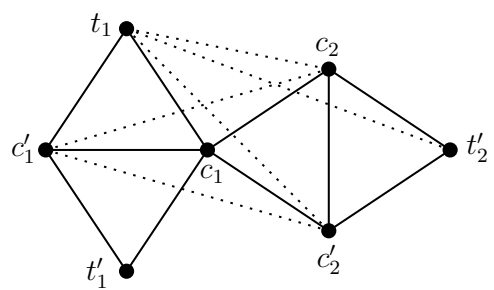

(a)

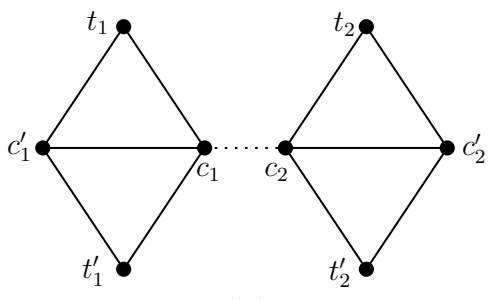

(b)

Figure 7: Two patterns $(V, E, F)$ used in the proofs. Graphs $(V, E)$ are depicted with solid lines. Possible additional edges (elements of $F$ ) are depicted with dotted lines.

We start with a lemma.

Lemma 7.1. Let $G$ be a connected chordal graph and let $H$ be an induced subgraph of $G$ that realizes the pattern in Figure 7(a). Moreover, suppose that:

(1) vertices $t_{1}$ and $t_{1}^{\prime}$ are in different components of $G-\left\{c_{1}, c_{1}^{\prime}\right\}$, and

(2) the component of $G-\left\{c_{1}, c_{1}^{\prime}\right\}$ containing $\left\{c_{2}, c_{2}^{\prime}, t_{2}^{\prime}\right\}$ has a vertex dominating $\left\{c_{1}, c_{1}^{\prime}\right\}$.

Then $G$ contains $F_{1}$ or $F_{2}$ as an induced subgraph.

Proof. By contradiction. Suppose that $G$ and $H$ satisfy the assumptions of the lemma, but $G$ is $\left\{F_{1}, F_{2}\right\}$-free. We first show that none of the dotted edges can be present in $H$. We infer that $c_{1}^{\prime} \nsim c_{2}$ and $c_{1}^{\prime} \nsim c_{2}^{\prime}$, for otherwise an induced $F_{1}$ or $F_{2}$ arises on the vertex set $V(H) \backslash\left\{t_{1}\right\}$, depending on whether one or both edges are present. Next, $t_{1} \nsim t_{2}^{\prime}$, since otherwise a 4 -cycle arises on the vertex set $\left\{t_{1}, c_{1}, c_{2}^{\prime}, t_{2}^{\prime}\right\}$ (if $t_{1} \nsim c_{2}^{\prime}$ ) or an induced $F_{1}$ 
arises on the vertex set $V(H) \backslash\left\{c_{2}\right\}$ (otherwise). Finally, we infer that $t_{1} \nsim c_{2}$ and $t_{1} \nsim c_{2}^{\prime}$, for otherwise an induced $F_{1}$ or $F_{2}$ arises on the vertex set $V(H) \backslash\left\{t_{1}^{\prime}\right\}$, depending whether one or both edges are present.

Let $K$ be the component of $G-\left\{c_{1}, c_{1}^{\prime}\right\}$ such that $V_{2}^{\prime}=\left\{c_{2}, c_{2}^{\prime}, t_{2}^{\prime}\right\} \subseteq V(K)$, and let $w \in V(K)$ be a vertex dominating $\left\{c_{1}, c_{1}^{\prime}\right\}$ that is closest to $V_{2}^{\prime}$ in $K$. The preceding paragraph implies that $w \notin V_{2}^{\prime}$. We will now show that $w \nsim v$ for any $v \in V_{2}^{\prime}$. Suppose for a contradiction that $w \sim v$ for some $v \in V_{2}^{\prime}$. Note that $w \notin\left\{t_{1}, t_{1}^{\prime}\right\}$ since there are no edges between the sets $\left\{t_{1}, t_{1}^{\prime}\right\}$ and $V_{2}^{\prime}$. Furthermore, property (1) implies that there exists some $t \in\left\{t_{1}, t_{1}^{\prime}\right\}$ such that $w \nsim t$. Suppose that $w \sim t_{2}^{\prime}$. Then $w \sim c_{2}$, since otherwise a 4 -cycle arises on the vertex set $\left\{w, c_{1}, c_{2}, t_{2}^{\prime}\right\}$. But now the vertex set $\left\{t_{2}^{\prime}, c_{2}, w, c_{1}, c_{1}^{\prime}, t\right\}$ induces a copy of $F_{1}$ in $G$. Therefore $w \nsim t_{2}^{\prime}$, and an induced $F_{1}$ or $F_{2}$ arises on the vertex set $V_{2}^{\prime} \cup\left\{w, c_{1}, c_{1}^{\prime}\right\}$, depending on whether $w$ is adjacent to one or both vertices in $\left\{c_{2}, c_{2}^{\prime}\right\}$. This contradiction shows that $w$ has no neighbor in $V_{2}^{\prime}$.

Let $P=\left(w=w_{1}, \ldots, w_{k}\right)$ with $w_{k} \in V_{2}^{\prime}$ be a shortest $w, V_{2}^{\prime}$-path in $K$. Note that $k \geq 3$ and the choice of $P$ implies that for all $i \in\{1, \ldots, k-2\}$ vertex $w_{i}$ is not adjacent to any vertex in $V_{2}^{\prime}$. In order to avoid an induced cycle of length at least 4 within $V(P) \cup V_{2}^{\prime} \cup\left\{c_{1}\right\}$, we infer that vertex $c_{1}$ must be adjacent to all the internal vertices of $P$ (that is, to $w_{2}, \ldots, w_{k-1}$ ). Next we infer that $w_{k-1} \sim t_{2}^{\prime}$, since otherwise the vertex set $V_{2}^{\prime} \cup\left\{c_{1}, w_{k-1}, w_{k-2}\right\}$ induces a copy of $F_{1}$ or $F_{2}$ (depending on the number of edges between $w_{k-1}$ and $\left\{c_{2}, c_{2}^{\prime}\right\}$ ). Moreover, to avoid an induced 4-cycle on the vertex set $\left\{t_{2}^{\prime}, w_{k-1}, c_{1}, c_{2}\right\}$, we infer that $w_{k-1} \sim c_{2}$. But now an induced $F_{1}$ arises on the vertex set $\left\{t_{2}^{\prime}, c_{2}, c_{1}, w_{k-1}, w_{k-2}, w_{k-3}\right\}$ (where if $k=3$ we define $w_{0}=c_{1}^{\prime}$ ). This last contradiction completes the proof of Lemma 7.1.

Let us now recall Lemma 5.3.

Lemma 5.3 (Diamond Lemma). Let $G$ be a connected chordal graph. Suppose that $G$ contains two induced diamonds $D_{1}=\left(V_{1}, E_{1}\right)$ and $D_{2}=\left(V_{2}, E_{2}\right)$ such that:

(i) $C_{1} \cap C_{2}=\emptyset$.

(ii) If no vertex in $C_{1}$ is adjacent to a vertex in $C_{2}$, then there exists a $C_{1}, C_{2}$-separator in $G$ of size one.

(iii) For each $j \in\{1,2\}$ the tips (i.e., $t_{j}, t_{j}^{\prime}$ ) of $D_{j}$ belong to different components of $G-C_{j}$.

(iv) For $j \in\{1,2\}$ every component of $G-C_{j}$ has a vertex that dominates $C_{j}$.

Then $G$ has an induced subgraph isomorphic to $F_{1}, F_{2}$, or $H_{i}$ for some $i \geq 1$, where the graphs $F_{1}, F_{2}$, and a general member of the family $\left\{H_{i}\right\}$ are depicted in Figure 3.

Proof. We will prove the Diamond Lemma by contradiction through a series of claims. Let $G$ be a connected chordal graph and let $D_{1}$ and $D_{2}$ be two induced diamonds with properties (i) - (iv) in $G$. Suppose for a contradiction that $G$ is $\left\{F_{1}, F_{2}, H_{1}, H_{2}, \ldots\right\}$-free.

Claim 1. For each $j \in\{1,2\}$, there exists some $t \in T_{j}$ such that $N[t] \cap C_{3-j}=\emptyset$ (that is, each diamond has a tip that is not adjacent to any center of the other diamond).

Proof. Suppose that each tip of $D_{j}$ is adjacent to at least one vertex in $C_{3-j}$. Then $T_{j}$ belongs to one component of $G-C_{j}$, contradicting property (iii). 
Claim 2. If there exists some $t \in T_{1} \cap T_{2}$, then $T_{1} \cap T_{2}=\{t\}$ and $T_{j} \cap C_{3-j}=\emptyset$ for $j \in\{1,2\}$.

Proof. Follows immediately from Claim 1 and property (iii).

Claim 3. $\left|V_{1} \cap V_{2}\right| \leq 1$.

Proof. First note that we have $\left|T_{1} \cap V_{2}\right| \leq 1$, since otherwise $T_{1}=T_{2}$, contradicting property (iii). Observe also that by property (i) we have $C_{1} \cap V_{2} \subseteq C_{1} \cap T_{2}$, implying that $\left|C_{1} \cap V_{2}\right| \leq 1$. Consequently $\left|V_{1} \cap V_{2}\right| \leq 2$.

Now suppose for a contradiction that $\left|V_{1} \cap V_{2}\right|=2$. By property (i) and Claim 2 we may assume without loss of generality that $c_{1}=t_{2}$ and $t_{1}^{\prime}=c_{2}^{\prime}$. To avoid an induced 4-cycle on the set $T_{1} \cup T_{2}$ we infer that $t_{1} \nsim t_{2}^{\prime}$. Furthermore, property (iii) implies that $c_{1}^{\prime} \nsim t_{2}^{\prime}$ and $c_{2} \nsim t_{1}$. But now the set $V_{1} \cup V_{2}$ induces a copy of $F_{1}$ (if $c_{1}^{\prime} \nsim c_{2}$ ) or a copy of $F_{2}$ (otherwise).

Claim 4. If $V_{1} \cap V_{2}=\{v\}$ then $v \in T_{1} \cap T_{2}$.

Proof. Suppose for a contradiction that $V_{1} \cap V_{2}=\{v\}$, and $v \notin T_{1} \cap T_{2}$. Property (i) implies that $v \in T_{j} \cap C_{3-j}$ for some $j \in\{1,2\}$, say $v=c_{1}=t_{2}$. Claim 1 implies (without loss of generality) that $t_{1}^{\prime} \nsim c_{2}$ and $t_{1}^{\prime} \nsim c_{2}^{\prime}$. Property (iii) implies that $c_{1}^{\prime} \nsim t_{2}^{\prime}$. Note that $t_{1}^{\prime} \nsim t_{2}^{\prime}$, for otherwise a 4 -cycle arises on the vertex set $\left\{t_{1}^{\prime}, c_{1}, c_{2}, t_{2}^{\prime}\right\}$. Now the subgraph of $G$ induced by $V_{1} \cup V_{2}$ realizes the pattern depicted in Figure 7(a) and we apply Lemma 7.1 to derive a contradiction.

Claim 5. $V_{1} \cap V_{2}=\emptyset$.

Proof. Suppose for a contradiction that $V_{1} \cap V_{2} \neq \emptyset$. Claim 3 implies that $V_{1} \cap V_{2}=\{v\}$ and by Claim 4, $v \in T_{1} \cap T_{2}$. Without loss of generality we may assume that $t_{1}=t_{2}$. Claim 1 implies that there is no edge between $t_{1}^{\prime}$ and $C_{2}$ and between $t_{2}^{\prime}$ and $C_{1}$. Furthermore, we must have $t_{1}^{\prime} \nsim t_{2}^{\prime}$ since otherwise $G$ contains an induced 4-cycle on the vertex set $\left\{t_{1}^{\prime}, c_{1}, c_{2}, t_{2}^{\prime}\right\}$ (if $c_{1} \sim c_{2}$ ) or an induced 5 -cycle on the vertex set $\left\{t_{1}^{\prime}, c_{1}, t_{1}, c_{2}, t_{2}^{\prime}\right\}$ (otherwise).

It remains to analyze the edges between $C_{1}$ and $C_{2}$. Clearly, $e\left(C_{1}, C_{2}\right) \in\{0,1, \ldots, 4\}$. Notice that

$$
e\left(C_{1}, C_{2}\right)= \begin{cases}0 & \text { implies an induced } H_{1} \text { on the set } V_{1} \cup V_{2} ; \\ 1 & \text { implies an induced } F_{1} \text { on the vertex set }\left(V_{1} \cup V_{2}\right) \backslash\left\{t_{1}^{\prime}\right\} ; \\ 3 & \text { implies an induced } F_{1} \text { on the vertex set }\left(V_{1} \cup V_{2}\right) \backslash\left\{t_{1}\right\} ; \\ 4 & \text { implies an induced } F_{2} \text { on the vertex set }\left(V_{1} \cup V_{2}\right) \backslash\left\{t_{1}\right\}\end{cases}
$$

Consequently $e\left(C_{1}, C_{2}\right)=2$, and without loss of generality, to avoid an induced 4-cycle, we may assume that $c_{1} \sim c_{2}$ and $c_{1} \sim c_{2}^{\prime}$. But now an induced $F_{2}$ arises on the vertex set $\left(V_{1} \cup V_{2}\right) \backslash\left\{t_{1}^{\prime}\right\}$.

In the rest of the proof of the Diamond Lemma we consider the edges between $V_{1}$ and $V_{2}$. By Claim 1 and property (iii) we may assume without loss of generality the following.

Assumption 1. $e\left(\left\{t_{1}^{\prime}\right\}, V_{2}\right)=e\left(\left\{t_{2}^{\prime}\right\}, V_{1}\right)=0$. 
Therefore, it remains to consider only the (non-)edges between $\left\{t_{1}\right\}$ and $C_{2}$, between $\left\{t_{2}\right\}$ and $C_{1}$, between $C_{1}$ and $C_{2}$, and between $\left\{t_{1}\right\}$ and $\left\{t_{2}\right\}$.

Claim 6. $e\left(C_{1}, C_{2}\right) \leq 1$.

Proof. Clearly, $e\left(C_{1}, C_{2}\right) \leq 4$. Note that if $e\left(C_{1}, C_{2}\right) \in\{3,4\}$, then the vertex set $\left(V_{1} \cup V_{2}\right) \backslash\left\{t_{1}, t_{2}\right\}$ induces either a copy of $F_{1}$ or a copy of $F_{2}$. Furthermore, if $e\left(C_{1}, C_{2}\right)=2$, then, to avoid an induced 4-cycle, we may assume without loss of generality that $c_{1} \sim c_{2}$ and $c_{1} \sim c_{2}^{\prime}$. Now the subgraph of $G$ induced by $\left(V_{1} \cup V_{2}\right) \backslash\left\{t_{2}\right\}$ realizes the pattern depicted in Figure $7(a)$ and we apply Lemma 7.1 to derive a contradiction.

By Claim 6 we may assume without loss of generality the following.

Assumption 2. $c_{1}^{\prime} \nsim c_{2}, c_{1}^{\prime} \nsim c_{2}^{\prime}$, and $c_{1} \nsim c_{2}^{\prime}$.

Claim 7. $e\left(\left\{t_{j}\right\}, C_{3-j}\right) \leq 1$ for $j \in\{1,2\}$.

Proof. Suppose for a contradiction that $e\left(t_{j}, C_{3-j}\right)=2$. To avoid an induced $H_{1}$ on the vertex set $\left(V_{1} \cup V_{2}\right) \backslash\left\{t_{3-j}\right\}$, we must have an edge between $C_{1}$ and $C_{2}$. By Claim 6 and Assumption 2, we have $c_{1} \sim c_{2}$, but now an induced $F_{1}$ arises on the vertex set $V_{j} \cup C_{3-j}$.

Claim 8. We may assume without loss of generality that $t_{j} \nsim c_{3-j}^{\prime}$ for $j \in\{1,2\}$.

Proof. Let $j \in\{1,2\}$. By Claim 7, we have that either $t_{j} \nsim c_{3-j}$ or $t_{j} \nsim c_{3-j}^{\prime}$. If both edges are missing, then there is nothing to show. Suppose now that $e\left(t_{j}, C_{3-j}\right)=1$. To see that we may assume that $t_{j} \sim c_{3-j}$, note that this can be achieved by swapping $c_{3-j}$ and $c_{3-j}^{\prime}$ (if necessary) when $c_{1} \nsim c_{2}$, while if $c_{1} \sim c_{2}$, then $t_{j} \sim c_{3-j}$, since otherwise the vertex set $\left\{t_{j}, c_{1}, c_{2}, c_{3-j}^{\prime}\right\}$ induces a 4 -cycle in $G$.

Claim 8 yields the following.

Assumption 3. $t_{1} \nsim c_{2}^{\prime}$ and $t_{2} \nsim c_{1}^{\prime}$.

Claim 9. $t_{1} \nsim t_{2}$.

Proof. Suppose for a contradiction that $t_{1} \sim t_{2}$. First we will show that $c_{1} \sim t_{2}$ or $c_{2} \sim t_{1}$. Suppose for a contradiction that $c_{1} \nsim t_{2}$, and $c_{2} \nsim t_{1}$. Then an induced $H_{2}$ arises on the set $V_{1} \cup V_{2}$ (if $c_{1} \nsim c_{2}$ ) or an induced 4-cycle on the vertex set $\left\{c_{1}, t_{1}, t_{2}, c_{2}\right\}$ (otherwise).

Without loss of generality we may assume that $c_{1} \sim t_{2}$. By Assumption 3 we have $t_{2} \nsim c_{1}^{\prime}$, and to avoid an induced $H_{1}$ on the vertex set $\left(V_{1} \cup V_{2}\right) \backslash\left\{t_{1}^{\prime}\right\}$, we must have an edge between $t_{1}$ and $C_{2}$ or $c_{1} \sim c_{2}$. If $t_{1} \sim c_{2}$, then the vertex set $C_{1} \cup C_{2} \cup\left\{t_{1}, t_{2}\right\}$ induces a copy of $F_{1}$ or $F_{2}$ (depending on whether $c_{1} \sim c_{2}$ or not). Consequently $t_{1} \nsim c_{2}$. Therefore the only edge we can have is $c_{1} \sim c_{2}$, but now an induced $F_{1}$ arises on the vertex set $C_{1} \cup C_{2} \cup\left\{t_{1}, t_{2}\right\}$.

Claim 10. $t_{1} \nsim c_{2}$ and $t_{2} \nsim c_{1}$. 
Proof. By symmetry, it suffices to show that $c_{1} \nsim t_{2}$. Suppose for a contradiction that $c_{1} \sim t_{2}$. Claim 9 implies that $t_{1} \nsim t_{2}$. Recall that by Assumption 1 we have $t_{2} \nsim t_{1}^{\prime}$. Furthermore $e\left(\left\{t_{1}\right\}, C_{2}\right)=0$, since otherwise $t_{1} \sim c_{2}$ (by Assumption 3) and either the vertex set $\left\{t_{1}, c_{1}, t_{2}, c_{2}\right\}$ induces a 4 -cycle (if $c_{1} \nsim c_{2}$ ) or the vertex set $C_{1} \cup C_{2} \cup\left\{t_{1}, t_{2}\right\}$ induces an $F_{1}$ (otherwise).

Let $K$ be the component of $G-C_{1}$ such that $V_{2} \subseteq V(K)$. By property (iv) there exists a vertex in $V(K)$ that dominates $C_{1}$. Let $w \in V(K)$ be a vertex that dominates $C_{1}$ and is closest to $V_{2}$ in $K$. Clearly, $w \notin V_{2}$. Property (iii) implies that there exists some $t \in T_{1}$ such that $w \neq t$ and $w \nsim t$. Note that $c_{1} \nsim c_{2}$, since otherwise the subgraph of $G$ induced by $C_{1} \cup C_{2} \cup\left\{w, t, t_{2}\right\}$ realizes the pattern depicted in Figure 7(a) and we apply Lemma 7.1 to derive a contradiction. Furthermore, $w \nsim t_{2}^{\prime}$, since otherwise $t_{2}$ and $t_{2}^{\prime}$ would belong to the same component of $G-C_{2}$, contradicting property (iii). Next, we have that $w \nsim c_{2}$, since otherwise either the vertex set $\left\{w, c_{1}, t_{2}, c_{2}\right\}$ induces a 4 -cycle (if $w \nsim t_{2}$ ) or the vertex set $C_{1} \cup\left\{t, w, t_{2}, c_{2}\right\}$ induces an $F_{1}$ (otherwise). By symmetry, $w \nsim c_{2}^{\prime}$. Consequently, $w \nsim t_{2}$, for otherwise a copy of $H_{1}$ arises on the vertex set $C_{1} \cup V_{2} \cup\{w\}$.

Let $P=\left(w=w_{1}, \ldots, w_{k}\right)$ with $w_{k} \in V_{2}$ be a shortest $w, V_{2}$-path in $K$. Note that $k \geq 3$ and that the choice of $P$ implies that for all $i \in\{1, \ldots, k-2\}$ vertex $w_{i}$ is not adjacent to any vertex in $V_{2}$. Furthermore, $w_{k-1} \nsim t_{2}^{\prime}$, since otherwise $t_{2}$ and $t_{2}^{\prime}$ would belong to the same component of $G-C_{2}$, contradicting property (iii). In order to avoid an induced cycle of length at least 4 within $V(P) \cup V_{2} \cup\left\{c_{1}\right\}$, we infer that vertex $c_{1}$ must be adjacent to all the internal vertices of $P$ (that is, $w_{2}, \ldots, w_{k-1}$ ). If $w_{k-1} \nsim t_{2}$, then $w_{k} \in C_{2}$, which yields an induced 4 -cycle on the vertex set $\left\{c_{1}, t_{2}, w_{k}, w_{k-1}\right\}$. Therefore, $w_{k-1} \sim t_{2}$. But now either an induced $H_{1}$ arises on the vertex set $V_{2} \cup\left\{w_{k-1}, w_{k-2}, c_{1}\right\}$ (if $e\left(\left\{w_{k-1}\right\}, C_{2}\right)=0$ ) or an induced $F_{1}$ or $F_{2}$ arises on the vertex set $V_{2} \cup\left\{w_{k-1}, c_{1}\right\}$ (otherwise).

Assumptions $1-3$ and Claims 7, 9, and 10 imply the following.

Claim 11. The only possible edge between $V_{1}$ and $V_{2}$ is the edge $c_{1} c_{2}$.

Let $H$ be the subgraph of $G$ induced by $V_{1} \cup V_{2}$. By Claim 11, $H$ realizes the pattern in Figure 7(b). Let $K_{2}^{-1}$ be the component of $G-C_{1}$ containing $V_{2}$ and let $U^{-1}$ be the set of vertices in $K_{2}^{-1}$ that dominate $C_{1}$. By property (iv), set $U^{-1}$ is non-empty. Let $u^{-1}$ be a vertex in $U^{-1}$ that is closest in $K_{2}^{-1}$ to $C_{2}$. Graph $K_{1}^{-2}$ and vertex $u^{-2}$ are defined analogously.

By property (iii) we may assume without loss of generality the following.

Assumption 4. $t_{1}^{\prime} \notin V\left(K_{2}^{-1}\right)$ and $t_{2}^{\prime} \notin V\left(K_{1}^{-2}\right)$.

Claim 12. $\left\{u^{-1}, u^{-2}\right\} \cap\left\{t_{1}^{\prime}, t_{2}^{\prime}\right\}=\emptyset$ and $e\left(\left\{u^{-1}, u^{-2}\right\},\left\{t_{1}^{\prime}, t_{2}^{\prime}\right\}\right)=0$.

Proof. Since $u^{-1} \in V\left(K_{2}^{-1}\right)$ and $t_{1}^{\prime} \notin V\left(K_{2}^{-1}\right)$, the definition of $K_{2}^{-1}$ implies that $u^{-1} \neq t_{1}^{\prime}$ and $u^{-1} \nsim t_{1}^{\prime}$. By symmetry, we also have $u^{-2} \neq t_{2}^{\prime}$ and $u^{-2} \nsim t_{2}^{\prime}$.

We next show that $u^{-1} \neq t_{2}^{\prime}$ and $u^{-1} \nsim t_{2}^{\prime}$ (and then the remaining inequality $u^{-2} \neq t_{1}^{\prime}$ and non-adjacency $u^{-2} \nsim t_{1}^{\prime}$ will follow by symmetry). First note that $u^{-1} \neq t_{2}^{\prime}$ since $u^{-1}$ dominates $C_{1}$ and $e\left(\left\{t_{2}^{\prime}\right\}, C_{1}\right)=0$ by Assumption 1. Suppose for a contradiction that $u^{-1} \sim t_{2}^{\prime}$. This implies that $u^{-1} \nsim t_{2}$, since otherwise $t_{2}$ and $t_{2}^{\prime}$ would belong to the same component of $G-C_{2}$, contradicting property (iii). But now, either an induced $\mathrm{H}_{2}$ arises on the vertex set $V_{2} \cup C_{1} \cup\left\{u^{-1}, t_{1}^{\prime}\right\}$ (if $e\left(\left\{u^{-1}\right\}, C_{2}\right)=0$ ), or an induced $H_{1}$ arises 
either on the vertex set $C_{1} \cup C_{2} \cup\left\{u^{-1}, t_{1}^{\prime}, t_{2}^{\prime}\right\}$ (if $e\left(\left\{u^{-1}\right\}, C_{2}\right)=1$ ) or on the vertex set $C_{1} \cup C_{2} \cup\left\{u^{-1}, t_{1}^{\prime}, t_{2}\right\}$ (otherwise).

Claim 13. Vertices $u^{-1}$ and $u^{-2}$ are distinct and non-adjacent, and at least one of the sets $N\left(u^{-1}\right) \cap V_{2}, N\left(u^{-2}\right) \cap V_{1}$ is empty.

Proof. First we prove that $u^{-1} \nsim c_{2}$ or $u^{-1} \nsim c_{2}^{\prime}$. Suppose for a contradiction that $e\left(\left\{u^{-1}\right\}, C_{2}\right)=2$. Then either an induced $F_{1}$ arises on the vertex set $C_{1} \cup C_{2} \cup\left\{u^{-1}, t_{2}^{\prime}\right\}$ (if $c_{1} \sim c_{2}$ ) or an induced $H_{1}$ arises on the vertex set $C_{1} \cup C_{2} \cup\left\{u^{-1}, t_{1}^{\prime}, t_{2}^{\prime}\right\}$ (otherwise). Therefore, $u^{-1} \nsim c_{2}$ or $u^{-1} \nsim c_{2}^{\prime}$, as claimed.

Since $u^{-2}$ dominates $C_{2}$ but $u^{-1}$ does not, we infer that $u^{-1} \neq u^{-2}$.

Next we prove that $u^{-1} \nsim u^{-2}$. Suppose for a contradiction that $u^{-1} \sim u^{-2}$. We claim that $u^{-1} \sim c_{2}$ or $u^{-2} \sim c_{1}$. Suppose to the contrary that $u^{-1} \nsim c_{2}$ and $u^{-2} \nsim c_{1}$. Then $c_{1} \nsim c_{2}$, since otherwise an induced 4-cycle arises on the vertex set $\left\{c_{1}, c_{2}, u^{-2}, u^{-1}\right\}$. Furthermore, $u^{-1} \sim c_{2}^{\prime}$ or $u^{-2} \sim c_{1}^{\prime}$, since otherwise an induced $H_{2}$ arises on the vertex set $C_{1} \cup C_{2} \cup\left\{t_{1}^{\prime}, u^{-1}, u^{-2}, t_{2}^{\prime}\right\}$. If only one of the edges $u^{-1} c_{2}^{\prime}$ and $u^{-2} c_{1}^{\prime}$ is present, say $u^{-1} c_{2}^{\prime}$, then an induced $H_{1}$ arises on the vertex set $C_{1} \cup C_{2} \cup\left\{t_{1}^{\prime}, u^{-1}, u^{-2}\right\}$. If both edges $u^{-1} c_{2}^{\prime}$ and $u^{-2} c_{1}^{\prime}$ are present, then an induced $F_{1}$ arises on the vertex set $C_{1} \cup C_{2} \cup\left\{u^{-1}, u^{-2}\right\}$. Both cases lead to a contradiction, thus $u^{-1} \sim c_{2}$ or $u^{-2} \sim c_{1}$, as claimed. We may assume without loss of generality that $u^{-1} \sim c_{2}$. Now we must have $c_{1} \nsim c_{2}$ and $c_{1} \nsim u^{-2}$, since otherwise an induced $F_{1}$ or $F_{2}$ arises on the vertex set $C_{1} \cup C_{2} \cup\left\{u^{-1}, u^{-2}\right\}$, depending on whether one or both edges are present. But now an induced $H_{1}$ arises on the vertex set $C_{1} \cup C_{2} \cup\left\{t_{1}^{\prime}, u^{-1}, u^{-2}\right\}$, a contradiction.

To complete the proof, we consider the two cases depending on whether $c_{1}$ is adjacent to $c_{2}$ or not. Suppose first that $c_{1} \sim c_{2}$. Then $u^{-1} \nsim c_{2}^{\prime}$, for otherwise $u^{-1} \nsim c_{2}$ and $G$ contains an induced 4 -cycle on the vertex set $\left\{u^{-1}, c_{2}^{\prime}, c_{2}, c_{1}\right\}$. By symmetry, we also have $u^{-2} \nsim c_{1}^{\prime}$. If $u^{-1} \sim c_{2}$ and $u^{-2} \sim c_{1}$, then an induced $F_{1}$ arises on the vertex set $C_{1} \cup C_{2} \cup\left\{u^{-1}, u^{-2}\right\}$. It follows that $H$ contains at most one of the edges $u^{-1} c_{2}$ and $u^{-2} c_{1}$. By symmetry, we may assume without loss of generality that $u^{-1} \nsim c_{2}$. We infer that $u^{-1} \nsim t_{2}$, since otherwise $G$ contains an induced $C_{4}$ on the vertex set $\left\{u^{-1}, c_{1}, c_{2}, t_{2}\right\}$. It follows that the set $N\left(u^{-1}\right) \cap V_{2}$ is empty.

Finally, suppose that $c_{1} \nsim c_{2}$. Then either $e\left(\left\{u^{-1}\right\}, C_{2}\right)=0$ or $e\left(\left\{u^{-2}\right\}, C_{1}\right)=0$, for otherwise $G$ contains an induced 4 -cycle on the vertex set $\left\{u^{-1}, x, u^{-2}, y\right\}$ where $x \in N\left(u^{-1}\right) \cap C_{2}$ and $y \in N\left(u^{-2}\right) \cap C_{1}$. By symmetry, we may assume without loss of generality that $e\left(\left\{u^{-1}\right\}, C_{2}\right)=0$. We infer that $u^{-1} \nsim t_{2}$, since otherwise $G$ contains an induced $H_{2}$ on the vertex set $C_{1} \cup V_{2} \cup\left\{u^{-1}, t_{1}^{\prime}\right\}$. It follows that the set $N\left(u^{-1}\right) \cap V_{2}$ is empty.

By Claim 13 we may assume without loss of generality the following.

Assumption 5. $e\left(\left\{u^{-1}\right\}, V_{2}\right)=0$.

Claim 14. $c_{1} \nsim c_{2}$.

Proof. Suppose for a contradiction that $c_{1} \sim c_{2}$ and consider $K_{2}^{-1}, u^{-1}, K_{1}^{-2}$, and $u^{-2}$. Clearly, $u^{-1} \notin C_{1} \cup C_{2} \cup\left\{t_{2}^{\prime}\right\}$. Moreover, by Claim 12 we have we have $u^{-1} \neq t_{1}^{\prime}$ and $u^{-1} \nsim t_{1}^{\prime}$. Also, by symmetry, $u^{-2} \notin C_{1} \cup C_{2} \cup\left\{t_{1}^{\prime}\right\}, u^{-2} \neq t_{2}^{\prime}$ and $u^{-2} \nsim t_{2}^{\prime}$. Furthermore, by Assumption 5 we have $N\left(u^{-1}\right) \cap V_{2}=\emptyset$.

Let $P^{-1}=\left(u^{-1}=u_{1}, u_{2}, \ldots, u_{k}\right)$, with $u_{k} \in V_{2}^{\prime}=C_{2} \cup\left\{t_{2}^{\prime}\right\}$ be a shortest $u^{-1}, V_{2}^{\prime}$-path in $K_{2}^{-1}$, and similarly, let $P^{-2}=\left(u^{-2}=v_{1}, v_{2}, \ldots, v_{\ell}\right)$, with $v_{\ell} \in V_{1}^{\prime}=$ 
$C_{1} \cup\left\{u^{-1}, t_{1}^{\prime}\right\}$ be a shortest $u^{-2}, V_{1}^{\prime}$-path in $V\left(K_{1}^{-2}\right)$. The fact that $N\left(u^{-1}\right) \cap V_{2}=\emptyset$ implies that $k \geq 3$. Furthermore, Claims 11 and 13 imply that $u^{-2} \notin V_{1} \cup\left\{u^{-1}\right\}$. Therefore, $\ell \geq 2$.

Since $u^{-1} \nsim c_{2}$, we infer that vertex $c_{1}$ must be adjacent to all the internal vertices of $P^{-1}$, for otherwise $G$ would contain an induced cycle of length at least 4 . Consequently, the definition of $u^{-1}$ implies that $u_{j} \nsim c_{1}^{\prime}$ for all $j \in\{2, \ldots, k-1\}$.

Suppose that $u_{k-1} \sim c_{2}^{\prime}$. To avoid an induced 4-cycle on the vertex set $\left\{c_{1}, c_{2}^{\prime}, c_{2}, u_{k-1}\right\}$, we infer that $u_{k-1} \sim c_{2}$. We must have $k=3$ since if $k \geq 4$, then the vertex set $C_{2} \cup\left\{c_{1}, u_{k-1}, u_{k-2}, u_{k-3}\right\}$ induces a copy of $F_{1}$. But now, since $c_{1}^{\prime} \nsim u_{2}$, an induced copy of $F_{1}$ arises on the vertex set $C_{1} \cup C_{2} \cup\left\{u_{1}, u_{2}\right\}$, a contradiction. Therefore, $u_{k-1} \nsim c_{2}^{\prime}$.

Suppose that $u_{k-1} \sim t_{2}^{\prime}$. To avoid an induced 4-cycle on the vertex set $\left\{c_{1}, c_{2}, t_{2}^{\prime}, u_{k-1}\right\}$, we must have $u_{k-1} \sim c_{2}$. But now, the vertex set $V_{2}^{\prime} \cup\left\{u_{k-1}, u_{k-2}, c_{1}\right\}$ induces a copy of $F_{1}$, a contradiction. Therefore, $u_{k-1} \nsim t_{2}^{\prime}$. Consequently, $u_{k}=c_{2}$.

Suppose that $u^{-2} \sim c_{1}$. If in addition $u^{-2} \nsim u_{k-1}$, then also $u^{-2} \nsim u_{k-2}$ (since otherwise the vertex set $\left\{u_{k-2}, u_{k-1}, c_{2}, u^{-2}\right\}$ would induce a 4 -cycle), but now, the vertex set $\left\{u_{k-2}, u_{k-1}, c_{1}, c_{2}, c_{2}^{\prime}, u^{-2}\right\}$ induces a copy of $F_{1}$, a contradiction. Therefore, $u^{-2} \sim u_{k-1}$. Let $u_{i}$ be the neighbor of $u^{-2}$ on $P^{-1}$ minimizing $i$. Since $u_{1} \nsim u^{-2}$, we have $i \geq 2$. Moreover, since $u^{-2} \sim u_{k-1}$, we have $i \leq k-1$. But now, the vertex set $C_{2} \cup\left\{u_{i-1}, c_{1}, u_{i}, u^{-2}\right\}$ induces either a copy of $F_{1}$ (if $u_{i} \nsim c_{2}$ ) or of $F_{2}$ (otherwise), a contradiction. Therefore, $u^{-2} \nsim c_{1}$.

Note that $N\left(u^{-2}\right) \cap V_{1}=\emptyset$, for otherwise if there is a vertex $x \in N\left(u^{-2}\right) \cap V_{1}$, then $x \neq c_{1}$ and $G$ contains an induced 4 -cycle on the vertex set $\left\{u^{-2}, c_{2}, c_{1}, x\right\}$, a contradiction. Since $N\left(u^{-2}\right) \cap V_{1}=\emptyset$, we can now apply symmetric arguments as for $P^{-1}$ to deduce that $\ell \geq 3$, vertex $c_{2}$ is adjacent to all the internal vertices of $P^{-2}$, and $v_{\ell}=c_{1}$.

Suppose first that $V\left(P^{-1}\right) \cap V\left(P^{-2}\right)=\emptyset$. To avoid an induced 4-cycle on the vertex set $\left\{u_{k-2}, c_{2}, c_{1}, v_{\ell-2}\right\}$, we infer that $u_{k-2} \nsim v_{\ell-2}$. Suppose that $u_{k-1} \nsim v_{\ell-1}$. Then also $u_{k-1} \nsim v_{\ell-2}$ (since otherwise we would have an induced 4-cycle on the vertex set $\left.\left\{u_{k-1}, v_{\ell-2}, v_{\ell-1}, c_{1}\right\}\right)$ and by a symmetric argument also $u_{k-2} \nsim v_{\ell-1}$. But now, we have an induced $F_{1}$ on the vertex set $\left\{u_{k-2}, c_{1}, u_{k-1}, c_{2}, v_{\ell-1}, v_{\ell-2}\right\}$. Thus, $u_{k-1} \sim v_{\ell-1}$. Moreover, we have either $u_{k-2} \sim v_{\ell-1}$ or $v_{\ell-2} \sim u_{k-1}$, since otherwise an induced $F_{2}$ arises on the vertex set $\left\{c_{1}, v_{\ell-1}, v_{\ell-2}, c_{2}, u_{k-1}, u_{k-2}\right\}$. Without loss of generality, assume that $u_{k-2} \sim v_{\ell-1}$. But now, setting $v_{0}=c_{2}^{\prime}$ if $\ell=3$, either an induced 4-cycle arises on the vertex set $\left\{u_{k-2}, v_{\ell-1}, v_{\ell-2}, v_{\ell-3}\right\}$ (if $u_{k-2} \sim v_{\ell-3}$ ) or an induced copy of $F_{1}$ arises on the vertex set $\left\{u_{k-2}, c_{1}, v_{\ell-1}, v_{\ell-2}, c_{2}, v_{\ell-3}\right\}$ (otherwise). This contradiction shows that $V\left(P^{-1}\right) \cap V\left(P^{-2}\right) \neq \emptyset$.

Since $v_{\ell}=c_{1}$ and due to the minimality of $P^{-2}$, we have $N\left(c_{1}\right) \cap V\left(P^{-2}\right)=\left\{v_{\ell-1}\right\}$. On the other hand, since $c_{1}$ dominates $P^{-1}$, we have $N\left(c_{1}\right) \cap V\left(P^{-1}\right)=V\left(P^{-1}\right)$. Therefore

$$
\begin{aligned}
\emptyset \neq V\left(P^{-2}\right) \cap V\left(P^{-1}\right)=V\left(P^{-2}\right) \cap\left(N\left(c_{1}\right) \cap V\left(P^{-1}\right)\right) \\
\quad=\left(N\left(c_{1}\right) \cap V\left(P^{-2}\right)\right) \cap V\left(P^{-1}\right)=\left\{v_{\ell-1}\right\} \cap V\left(P^{-1}\right) \subseteq\left\{v_{\ell-1}\right\},
\end{aligned}
$$

which yields $V\left(P^{-1}\right) \cap V\left(P^{-2}\right)=\left\{v_{\ell-1}\right\}$. A symmetric argument implies that $V\left(P^{-1}\right) \cap V\left(P^{-2}\right)=\left\{u_{k-1}\right\}$; in particular, $v_{\ell-1}=u_{k-1}$. To avoid an induced 4-cycle on the vertex set $\left\{u_{k-2}, c_{1}, c_{2}, v_{\ell-2}\right\}$, we infer that $u_{k-2} \nsim v_{\ell-2}$. But now, an induced copy of $F_{1}$ arises on the vertex set $\left\{u_{k-3}, u_{k-2}, c_{1}, u_{k-1}, c_{2}, v_{\ell-2}\right\}$ (where if $k=3$ we define $u_{0}=c_{1}^{\prime}$ ). This contradiction completes the proof of Claim 14 . 
By Claim 5, we have $V_{1} \cap V_{2}=\emptyset$. By Assumptions 1 and 2 and Claims 9, 10, and 14 we have $e\left(V_{1}, V_{2}\right)=0$. However, since $G$ is connected, there exists a path connecting the two diamonds $D_{1}$ and $D_{2}$. In particular, we will again consider $K_{2}^{-1}, u^{-1}, K_{1}^{-2}$, and $u^{-2}$, and analyze the possible interrelations between two particular paths to produce a forbidden induced subgraph.

Recall that by Assumption 4 we have $t_{1}^{\prime} \notin V\left(K_{2}^{-1}\right)$ and $t_{2}^{\prime} \notin V\left(K_{1}^{-2}\right)$. Furthermore, since $e\left(V_{1}, V_{2}\right)=0$, we have $u^{-1} \notin V_{2}$ and $u^{-2} \notin V_{1}$. Recall also that Claim 13 implies that $u^{-1} \neq u^{-2}, u^{-1} \nsim u^{-2}$.

Let $P^{-1}=\left(u^{-1}=u_{1}, u_{2}, \ldots, u_{k}\right)$, with $u_{k} \in C_{2}$, be a shortest $u^{-1}, C_{2}$-path in $K_{2}^{-1}$, and let $P^{-2}=\left(u^{-2}=v_{1}, v_{2}, \ldots, v_{\ell}\right)$, with $v_{\ell} \in C_{1}$, be a shortest $u^{-2}, C_{1}$-path in $K_{1}^{-2}$. We may assume that $u_{k}=c_{2}$ and $v_{\ell}=c_{1}$. The fact that $N\left(u^{-1}\right) \cap V_{2}=\emptyset$ implies that $k \geq 3$ and since $u^{-2} \notin C_{1}$, we have $\ell \geq 2$.

Claim 15. $\ell \geq 3$.

Proof. Suppose that $\ell=2$. Then, $u^{-2} \sim c_{1}$. Moreover, we have that $u^{-2} \nsim c_{1}^{\prime}$ since otherwise $u^{-2}$ would be a vertex in $U^{-1}$ closer in $K_{2}^{-1}$ to $C_{2}$ than $u^{-1}$, which is impossible due to the definition of $u^{-1}$.

We first show that $u^{-2} \neq u_{k-1}$. Suppose that $u^{-2}=u_{k-1}$. Then $u_{k-1} \sim c_{1}$ and $u_{k-1} \sim c_{2}^{\prime}$. Hence, in order to avoid an induced cycle of length at least 4 within $V\left(P^{-1}\right) \cup\left\{c_{1}\right\}$, we infer that vertex $c_{1}$ must be adjacent to all the internal vertices of $P^{-1}$. By Assumption 4, vertex $t_{2}^{\prime}$ has no neighbors in the set $V\left(K_{1}^{-2}\right)$; in particular, $t_{2}^{\prime}$ has no neighbors in the set $V\left(P^{-1}\right) \cup C_{1}$. Therefore, $G$ contains an induced $H_{1}$ on the vertex set $C_{2} \cup\left\{t_{2}^{\prime}, c_{1}, u_{k-1}, u_{k-2}, u_{k-3}\right\}$ (where if $k=3$ we define $u_{0}=c_{1}^{\prime}$ ), a contradiction.

Suppose that $u_{k-1} \sim c_{1}$. In particular, $u_{k-1} \neq t_{2}^{\prime}$. To avoid an induced 4-cycle on the vertex set $\left\{c_{1}, u_{k-1}, c_{2}, u^{-2}\right\}$, we infer that $u_{k-1} \sim u^{-2}$. Moreover, $u_{k-1} \sim t_{2}^{\prime}$ since otherwise the vertex set $C_{2} \cup\left\{t_{2}^{\prime}, u^{-2}, u_{k-1}, c_{1}\right\}$ induces a copy of either $F_{1}$ (if $u_{k-1} \nsim c_{2}^{\prime}$ ) or $F_{2}$ (otherwise). But now $u^{-2}$ and $t_{2}^{\prime}$ are in the same component of $G-C_{2}$, contradicting the fact that $u^{-2} \in V\left(K_{1}^{-2}\right)$ and $t_{2}^{\prime} \notin V\left(K_{1}^{-2}\right)$. This contradiction implies that $u_{k-1} \nsim c_{1}$.

Let $j \in\{1, \ldots, k\}$ be the maximum index such that $c_{1} \sim u_{j}$. Then $j \leq k-2$. To avoid a long induced cycle, we infer that $c_{1} \sim u_{j^{\prime}}$ for all $j^{\prime} \in\{1, \ldots, j\}$. Let $i \in\{1, \ldots, k\}$ be the minimum index such that $u^{-2} \sim u_{i}$. Note that $i>1$ since $u_{1}=u^{-1} \nsim u^{-2}$. To avoid a long induced cycle, we infer that $i \leq j$ and that $u^{-2} \sim u_{i^{\prime}}$ for all $i^{\prime} \in\{i, \ldots, k\}$. Note that if $i<j$, then $\left(u^{-1}=u_{1}, u_{2}, \ldots, u_{i}, u^{-2}, u_{k}=c_{2}\right)$ is a $u^{-1}, V_{2}^{\prime}$-path in $K_{2}^{-1}$ strictly shorter than $P^{-1}$, contradicting the minimality of $P^{-1}$. Therefore, $i=j$. But now, the vertex set $\left\{u_{j-1}, u_{j}, u_{j+1}, u_{j+2}, u^{-2}, c_{1}\right\}$ induces a copy of $F_{1}$. This contradiction implies that $\ell \geq 3$.

Claim 16. $u_{k-1} \neq v_{1}$ and $v_{\ell-1} \neq u_{1}$.

Proof. Suppose for a contradiction that $u_{k-1}=v_{1}$. Recall that $v_{1}=u^{-2}$. By the minimality of $P^{-1}$, we have $c_{2} \nsim u_{j}$ and $c_{2}^{\prime} \nsim u_{j}$ for every $j \in\{1, \ldots, k-2\}$. Furthermore, since $u_{1}=u^{-1} \nsim u^{-2}=u_{k-1}$, we have $k \geq 4$. Since $u^{-2}$ and $t_{2}^{\prime}$ are in different components of $G-C_{2}$, we infer that $t_{2}^{\prime} \nsim u_{j}$ for all $j \in\{1, \ldots, k-2\}$. If $c_{1} \sim u_{3}$, then we obtain an induced copy of $H_{i}$ for some $i \geq 1$ on the vertex set

$$
C_{2} \cup\left\{t_{2}^{\prime}, v_{1}=u_{k-1}, u_{k-2}, \ldots, u_{j}, u_{j-1}, u_{j-2}, c_{1}\right\},
$$

where $j \in\{3, \ldots, k\}$ is the maximum index such that $c_{1} \sim u_{j}$. (Note that $j \leq k-2$ since $c_{1} \nsim c_{2}=u_{k}$ and $c_{1}=v_{\ell} \nsim v_{1}=u_{k-1}$ by Claim 15.) 
Therefore, $c_{1} \nsim u_{3}$, and to avoid a long induced cycle, also $c_{1} \nsim u_{j}$ for $j \geq 4$. A similar argument shows that $c_{1}^{\prime} \nsim u_{j}$ for $j \geq 3$. If $c_{1} \nsim u_{2}$ and $c_{1}^{\prime} \nsim u_{2}$, then we obtain an induced copy of some $H_{i}$ on the vertex set $V\left(P^{-1}\right) \cup C_{1} \cup C_{2} \cup\left\{t_{1}^{\prime}, t_{2}^{\prime}\right\}$. If $c_{1} \sim u_{2}$ and $c_{1}^{\prime} \nsim u_{2}$ (or vice-versa), then an induced copy of some $H_{i}$ arises on the vertex set $V\left(P^{-1}\right) \cup C_{1} \cup C_{2} \cup\left\{t_{2}^{\prime}\right\}$, and if $c_{1} \sim u_{2}$ and $c_{1}^{\prime} \sim u_{2}$, then an induced copy of some $H_{i}$ arises on the vertex set $\left(V\left(P^{-1}\right) \backslash\left\{u_{1}\right\}\right) \cup C_{1} \cup C_{2} \cup\left\{t_{1}^{\prime}, t_{2}^{\prime}\right\}$. This contradiction shows that $u_{k-1} \neq v_{1}$.

Similar arguments as above imply that $v_{\ell-1} \neq u_{1}$.

Property (ii) in the statement of the Diamond Lemma implies the following.

Claim 17. $V\left(P^{-1}\right) \cap V\left(P^{-2}\right) \neq \emptyset$.

We are now ready to complete the proof of the Diamond Lemma. Let $r \in\{1, \ldots, k\}$ be the minimum index such that $u_{r} \in V\left(P^{-2}\right)$. Note that $r<k$, since $u_{k} \in C_{2}$ and $C_{2} \cap V\left(P^{-2}\right)=\emptyset$. Let $s \in\{1, \ldots, \ell\}$ be the index such that $u_{r}=v_{s}$. If $r=1$, then $u_{1}=v_{\ell-1}$, contradicting Claim 16. Therefore, $r \geq 2$. Similarly, if $s=1$, then $v_{1}=u_{k-1}$, again contradicting Claim 16. Therefore, $s \geq 2$.

Consider the path $Q=\left(u_{1}, \ldots, u_{r}=v_{s}, v_{s-1}, \ldots, v_{1}\right)$. Let $D$ and $D^{\prime}$ be the subgraphs of $G$ induced by $\left\{t_{1}^{\prime}, c_{1}, c_{1}^{\prime}, u_{1}\right\}$ and $\left\{t_{2}^{\prime}, c_{2}, c_{2}^{\prime}, v_{1}\right\}$, respectively. Notice that $D$ and $D^{\prime}$ are diamonds. We will refer to tips $u_{1}$ and $v_{1}$ as the roots of $D$ and $D^{\prime}$, respectively. Then, $Q$ is a path connecting the two roots. Moreover, by Assumption 4 we have $t_{1}^{\prime} \notin V\left(K_{2}^{-1}\right)$ and $V(Q) \subseteq V\left(K_{2}^{-1}\right)$, we infer that $t_{1}^{\prime}$ has no neighbors on $Q$. Similarly, $t_{2}^{\prime}$ has no neighbors on $Q$.

We may also assume that $Q$ is an induced path; otherwise, we replace $Q$ with a shortest $u_{1}, v_{1}$-path in $G[V(Q)]$. To complete the proof, we will show that $G$ is not $\left\{F_{1}, F_{2}, H_{1}\right.$, $\left.H_{2}, \ldots\right\}$-free. We say that an induced subgraph $H$ of $G$ is a weakly induced $H_{n}$ if $H$ has a spanning subgraph $H_{n}$ with $n \geq 1$ consisting of two diamonds and a path connecting them such that, assuming notation from Figure 8, the following holds:

(i) each of the two diamonds is induced in $G$,

(ii) there are no edges in $G$ connecting a vertex from one diamond with a vertex from another diamond, except perhaps edges incident with their roots (if $n=1$ ) or the unique edge on the path connecting the two roots (if $n=2$ ),

(iii) the path connecting the two diamonds is induced in $G$, and

(iv) vertices $x_{1}$ and $z_{1}$ do not have any neighbors on the path.

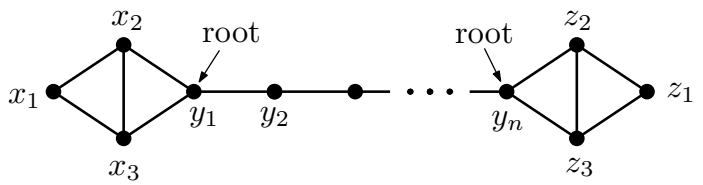

Figure 8: A weakly induced $H_{n}$.

Note, in particular, that for $n \in\{1,2\}$ every weakly induced $H_{n}$ is isomorphic to $H_{n}$. 
The above considerations show that the subgraph of $G$ induced by

$$
V(D) \cup V\left(D^{\prime}\right) \cup V(Q)
$$

contains a weakly induced $H_{n}$. Choose one such induced subgraph, say $H$, with minimum value of $n$, and let $F$ be the corresponding spanning subgraph of $H$ isomorphic to $H_{n}$. To complete the proof, we will now show that either $H$ equals $F$ or $G$ contains an induced $F_{1}$ or $F_{2}$. Suppose that this is not the case. The only possible edges that can be present in $H$ but not in $F$ are those connecting one of the vertices $x_{2}, x_{3}, z_{2}, z_{3}$ with one of the vertices in the set $\left\{y_{2}, \ldots, y_{n-1}\right\}$.

Let us first show that for each $i \in\{2, \ldots, n-1\}$, at most one of $x_{2}$ and $x_{3}$ is adjacent to $y_{i}$. Suppose that $x_{2} \sim y_{i}$ and $x_{3} \sim y_{i}$ for some $i \in\{2, \ldots, n-1\}$. Then $y_{i} \sim z_{2}$ or $y_{i} \sim z_{3}$, since otherwise the subgraph of $G$ induced by $\left\{x_{1}, x_{2}, x_{3}, y_{i}, \ldots, y_{n}, z_{1}, z_{2}, z_{3}\right\}$ would be a weakly induced $H_{n-i+1}$, contradicting the minimality of $H$. If $y_{i} \sim z_{2}$ and $y_{i} \sim z_{3}$, then the vertex set $\left\{x_{1}, x_{2}, x_{3}, y_{i}, z_{1}, z_{2}, z_{3}\right\}$ induces an $H_{1}$ in $G$. We may thus assume that $y_{i}$ is adjacent only to one of $z_{2}, z_{3}$, say to $z_{3}$. If $i=n-1$, then the vertex set $\left\{x_{1}, x_{2}, x_{3}, y_{n-1}, y_{n}, z_{2}, z_{3}\right\}$ induces an $H_{1}$ in $G$. If $i \leq n-2$, then the fact that $G$ is chordal implies that $z_{3} \sim y_{j}$ for all $j \in\{i, \ldots, n\}$, and the vertex set $\left\{x_{1}, x_{2}, x_{3}, y_{i}, y_{i+1}, y_{i+2}, z_{3}\right\}$ induces an $H_{1}$ in $G$. This contradiction shows that for each $i \in\{2, \ldots, n-1\}$, at most one of $x_{2}$ and $x_{3}$ is adjacent to $y_{i}$.

Next, we argue that at least one of $x_{2}$ and $x_{3}$ is not adjacent to any vertex $y_{i}$ with $i \in\{2, \ldots, n-1\}$. Indeed, if $x_{2} \sim y_{r}$ and $x_{3} \sim y_{s}$, with $2 \leq r \leq s \leq n-1$ (say), then $r<s$ and the fact that $G$ is chordal implies that $x_{3} \sim y_{j}$ for all $j \in\{2, \ldots, s\}$, contradicting the fact that at most one of $x_{2}$ and $x_{3}$ is adjacent to $y_{r}$. Therefore, we may assume without loss of generality that $x_{2}$ has no neighbors in the set $\left\{y_{2}, \ldots, y_{n-1}\right\}$. Similarly, we may assume that $z_{2}$ has no neighbors in the set $\left\{y_{2}, \ldots, y_{n-1}\right\}$.

Let $r \in\{1, \ldots, n-1\}$ be the maximum index such that $x_{3} \sim y_{r}$. Similarly, let $s \in\{2, \ldots, n\}$ be the minimum index such that $z_{3} \sim y_{s}$. If $r=1$ and $s=n$, then $H=F$ and we are done. Thus, we may assume without loss of generality that $r \geq 2$. In particular, this implies that $x_{3} \sim y_{2}$ (since $G$ is chordal). If $y_{2} \nsim z_{3}$, then the subgraph of $G$ induced by $\left\{x_{2}, x_{3}, y_{1}, \ldots, y_{n}, z_{1}, z_{2}, z_{3}\right\}$ is a weakly induced $H_{n-1}$, contradicting the minimality of $n$. Therefore, $y_{2} \sim z_{3}$, or, equivalently, $s=2$. A similar argument shows that $r=n-1$. Now, if $n=3$, then the vertex set $\left\{x_{2}, x_{3}, y_{1}, y_{2}, y_{3}, z_{2}, z_{3}\right\}$ induces an $H_{1}$ in $G$, and if $n \geq 4$, then the vertex set $\left\{x_{2}, x_{3}, y_{1}, y_{2}, y_{3}, z_{3}\right\}$ induces an $F_{1}$ in $G$. This contradiction completes the proof of the Diamond Lemma.

\section{References}

[1] C. Benzaken and P. L. Hammer, Linear separation of dominating sets in graphs, Ann. Discrete Math. 3 (1978), 1-10, doi:10.1016/s0167-5060(08)70492-8.

[2] C. Berge, Hypergraphs, volume 45 of North-Holland Mathematical Library, North-Holland, Amsterdam, 1989.

[3] A. Berry, J.-P. Bordat and O. Cogis, Generating all the minimal separators of a graph, Internat. J. Found. Comput. Sci. 11 (2000), 397-403, doi:10.1142/s0129054100000211.

[4] A. Berry and R. Pogorelcnik, A simple algorithm to generate the minimal separators and the maximal cliques of a chordal graph, Inform. Process. Lett. 111 (2011), 508-511, doi:10.1016/ j.ipl.2011.02.013. 
[5] P. Bertolazzi and A. Sassano, An $O(m n)$ algorithm for regular set-covering problems, Theoret. Comput. Sci. 54 (1987), 237-247, doi:10.1016/0304-3975(87)90131-9.

[6] J. Blum, M. Ding, A. Thaeler and X. Cheng, Connected dominating set in sensor networks and MANETs, in: D.-Z. Du and P. M. Pardalos (eds.), Handbook of Combinatorial Optimization, Supplement Volume B, Springer, New York, pp. 329-369, 2005, doi:10.1007/0-387-23830-1_8.

[7] E. Boros, Dualization of Aligned Boolean Functions, RUTCOR Research Report 9-94, Rutgers Center for Operations Research, 1994, http: / / rutcor. rutgers . edu/pub/rrr/ reports94/09.ps.

[8] E. Boros, V. Gurvich and M. Milanič, Decomposing 1-Sperner hypergraphs, 2018, arXiv:1510.02438 [math.CO].

[9] V. Bouchitté and I. Todinca, Treewidth and minimum fill-in: grouping the minimal separators, SIAM J. Comput. 31 (2001), 212-232, doi:10.1137/s0097539799359683.

[10] A. Brandstädt, V. B. Le and J. P. Spinrad, Graph Classes: A Survey, SIAM Monographs on Discrete Mathematics and Applications, SIAM, Philadelphia, Pennsylvania, 1999, doi: 10.1137/1.9780898719796.

[11] S. Butenko, S. Kahruman-Anderoglu and O. Ursulenko, On connected domination in unit ball graphs, Optim. Lett. 5 (2011), 195-205, doi:10.1007/s11590-010-0211-0.

[12] L. S. Chandran, A. Das, D. Rajendraprasad and N. M. Varma, Rainbow connection number and connected dominating sets, J. Graph Theory 71 (2012), 206-218, doi:10.1002/jgt.20643.

[13] L. S. Chandran and F. Grandoni, A linear time algorithm to list the minimal separators of chordal graphs, Discrete Math. 306 (2006), 351-358, doi:10.1016/j.disc.2005.12.010.

[14] M.-S. Chang, Weighted domination of cocomparability graphs, Discrete Appl. Math. 80 (1997), 135-148, doi:10.1016/s0166-218x(97)80001-7.

[15] M.-S. Chang, Efficient algorithms for the domination problems on interval and circular-arc graphs, SIAM J. Comput. 27 (1998), 1671-1694, doi:10.1137/s0097539792238431.

[16] N. Chiarelli and M. Milanič, Linear separation of total dominating sets in graphs, in: K. Jansen and R. Reischuk (eds.), Graph-Theoretic Concepts in Computer Science, Springer, Heidelberg, volume 8165 of Lecture Notes in Computer Science, pp. 165-176, 2013, doi:10.1007/ 978-3-642-45043-3_15, revised papers from the 39th International Workshop (WG 2013) held in Lübeck, June $19-21,2013$.

[17] N. Chiarelli and M. Milanič, Linear separation of connected dominating sets in graphs (extended abstract), in: International Symposium on Artificial Intelligence and Mathematics (ISAIM 2014), 2014, held in Fort Lauderdale, Florida, USA, January 6 - 8, 2014, https://www.cs.uic.edu/pub/Isaim2014/WebPreferences/ ISAIM2014_Boolean_Chiarelli_Milanic.pdf.

[18] N. Chiarelli and M. Milanič, Total domishold graphs: a generalization of threshold graphs, with connections to threshold hypergraphs, Discrete Appl. Math. 179 (2014), 1-12, doi:10.1016/j. dam.2014.09.001.

[19] C. K. Chow, Boolean functions realizable with single threshold devices, Proc. IRE 49 (1961), 370-371, doi:10.1109/jrproc.1961.287827.

[20] V. Chvátal and P. L. Hammer, Aggregation of inequalities in integer programming, in: P. L. Hammer, E. L. Johnson, B. H. Korte and G. L. Nemhauser (eds.), Studies in Integer Programming, North-Holland, Amsterdam, volume 1 of Annals of Discrete Mathematics, 1977 pp. 145-162, doi:10.1016/s0167-5060(08)70731-3, proceedings of the Workshop on Integer Programming held in Bonn, September 8 - 12, 1975. 
[21] Y. Crama, Dualization of regular Boolean functions, Discrete Appl. Math. 16 (1987), 79-85, doi:10.1016/0166-218x(87)90056-4.

[22] Y. Crama and P. L. Hammer, Boolean Functions: Theory, Algorithms, and Applications, volume 142 of Encyclopedia of Mathematics and its Applications, Cambridge University Press, Cambridge, 2011, doi:10.1017/cbo9780511852008.

[23] H. N. de Ridder et al., Information System on Graph Classes and their Inclusions (ISGCI), 2001-2014, http: / / www. graphclasses.org/ (accessed on 30 August 2016).

[24] I. Dinur and D. Steurer, Analytical approach to parallel repetition, in: D. Shmoys (ed.), STOC'14: Proceedings of the 2014 ACM Symposium on Theory of Computing, ACM Press, New York, pp. 624-633, 2014, held in New York, May 31 - June 3, 2014.

[25] G. A. Dirac, On rigid circuit graphs, Abh. Math. Sem. Univ. Hamburg 25 (1961), 71-76, doi: 10.1007/bf02992776.

[26] D.-Z. Du and P.-J. Wan, Connected Dominating Set: Theory and Applications, volume 77 of Springer Optimization and Its Applications, Springer, New York, 2013, doi:10.1007/ 978-1-4614-5242-3.

[27] W. Duckworth and B. Mans, Connected domination of regular graphs, Discrete Math. 309 (2009), 2305-2322, doi:10.1016/j.disc.2008.05.029.

[28] T. Eiter, K. Makino and G. Gottlob, Computational aspects of monotone dualization: a brief survey, Discrete Appl. Math. 156 (2008), 2035-2049, doi:10.1016/j.dam.2007.04.017.

[29] C. C. Elgot, Truth functions realizable by single threshold organs, in: Switching Circuit Theory and Logical Design, IEEE Computer Society, 1961 pp. 225-245, doi:10.1109/focs.1961.39, papers from the 1st Annual Symposium held in Chicago, Illinois, USA, October 9-14, 1960.

[30] S. Foldes and P. L. Hammer, Split graphs, in: F. Hoffman, R. C. Mullin, K. B. Reid and R. G. Stanton (eds.), Proceedings of the Eighth Southeastern Conference on Combinatorics, Graph Theory and Computing, Utilitas Mathematica Publishing, Winnipeg, Manitoba, 1977 pp. 311315, held at Louisiana State University, Baton Rouge, Louisiana, February 28 - March 3, 1977.

[31] F. V. Fomin, F. Grandoni and D. Kratsch, Solving connected dominating set faster than $2^{n}$, Algorithmica 52 (2008), 153-166, doi:10.1007/s00453-007-9145-z.

[32] I. J. Gabelman, The Functional Behavior of Majority (Threshold) Elements, Ph.D. thesis, Department of Electrical Engineering, Syracuse University, New York, 1961.

[33] M. C. Golumbic, Trivially perfect graphs, Discrete Math. 24 (1978), 105-107, doi:10.1016/ 0012-365x(78)90178-4.

[34] M. C. Golumbic, Algorithmic Graph Theory and Perfect Graphs, volume 57 of Annals of Discrete Mathematics, Elsevier, Amsterdam, 2nd edition, 2004, doi:10.1016/c2013-0-10739-8.

[35] T. W. Haynes, S. T. Hedetniemi and P. J. Slater (eds.), Domination in Graphs: Advanced Topics, volume 209 of Monographs and Textbooks in Pure and Applied Mathematics, Marcel Dekker, New York, 1998.

[36] T. W. Haynes, S. T. Hedetniemi and P. J. Slater, Fundamentals of Domination in Graphs, volume 208 of Monographs and Textbooks in Pure and Applied Mathematics, Marcel Dekker, New York, 1998.

[37] R.-W. Hung and M.-S. Chang, A simple linear algorithm for the connected domination problem in circular-arc graphs, Discuss. Math. Graph Theory 24 (2004), 137-145, doi:10.7151/dmgt. 1220.

[38] M. M. Kanté, V. Limouzy, A. Mary and L. Nourine, On the enumeration of minimal dominating sets and related notions, SIAM J. Discrete Math. 28 (2014), 1916-1929, doi:10.1137/ 120862612 . 
[39] N. Karmarkar, A new polynomial-time algorithm for linear programming, Combinatorica 4 (1984), 373-395, doi:10.1007/bf02579150.

[40] J. M. Keil, The complexity of domination problems in circle graphs, Discrete Appl. Math. 42 (1993), 51-63, doi:10.1016/0166-218x(93)90178-q.

[41] H. Kellerer, U. Pferschy and D. Pisinger, Knapsack Problems, Springer-Verlag, Berlin, 2004, doi:10.1007/978-3-540-24777-7.

[42] C. J. Klivans, Threshold graphs, shifted complexes, and graphical complexes, Discrete Math. 307 (2007), 2591-2597, doi:10.1016/j.disc.2006.11.018.

[43] T. Kloks, H. Bodlaender, H. Müller and D. Kratsch, Computing treewidth and minimum fillin: all you need are the minimal separators, in: T. Lengauer (ed.), Algorithms - ESA '93, Springer, Berlin, volume 726 of Lecture Notes in Computer Science, 1993 pp. 260-271, doi: 10.1007/3-540-57273-2_61, proceedings of the First Annual European Symposium held in Bad Honnef, September 30 - October 2, 1993.

[44] T. Kloks and D. Kratsch, Listing all minimal separators of a graph, SIAM J. Comput. 27 (1998), 605-613, doi:10.1137/s009753979427087x.

[45] P. S. Kumar and C. E. Veni Madhavan, Minimal vertex separators of chordal graphs, Discrete Appl. Math. 89 (1998), 155-168, doi:10.1016/s0166-218x(98)00123-1.

[46] R. Laskar and J. Pfaff, Domination and Irredundance in Split Graphs, Technical Report 430, Department of Mathematical Sciences, Clemson University, Clemson, South Carolina, 1983.

[47] V. E. Levit, M. Milanič and D. Tankus, On the recognition of $k$-equistable graphs, in: M. C. Golumbic, M. Stern, A. Levy and G. Morgenstern (eds.), Graph-Theoretic Concepts in Computer Science, Springer, Heidelberg, volume 7551 of Lecture Notes in Computer Science, pp. 286-296, 2012, doi:10.1007/978-3-642-34611-8_29, revised selected papers from the 38th International Workshop (WG 2012) held in Jerusalem, June 26 - 28, 2012.

[48] N. V. R. Mahadev and U. N. Peled, Threshold Graphs and Related Topics, volume 56 of Annals of Discrete Mathematics, North-Holland, Amsterdam, 1995.

[49] D. Michalak, Hereditarily dominated graphs, Discrete Math. 307 (2007), 952-957, doi:10. 1016/j.disc.2005.11.044.

[50] M. Milanič, J. Orlin and G. Rudolf, Complexity results for equistable graphs and related classes, Ann. Oper. Res. 188 (2011), 359-370, doi:10.1007/s10479-010-0720-3.

[51] P. Montealegre and I. Todinca, On distance- $d$ independent set and other problems in graphs with "few" minimal separators, in: P. Heggernes (ed.), Graph-Theoretic Concepts in Computer Science, Springer, Berlin, volume 9941 of Lecture Notes in Computer Science, pp. 183-194, 2016, doi:10.1007/978-3-662-53536-3_16, revised selected papers from the 42nd International Workshop (WG 2016) held at Boğaziçi University, Istanbul, June 22 - 24, 2016.

[52] H. Müller and A. Brandstädt, The NP-completeness of steiner tree and dominating set for chordal bipartite graphs, Theoret. Comput. Sci. 53 (1987), 257-265, doi:10.1016/ 0304-3975(87)90067-3.

[53] S. Muroga, Threshold Logic and its Applications, Wiley-Interscience (John Wiley \& Sons), New York-London-Sydney, 1971.

[54] C. Payan, A class of threshold and domishold graphs: equistable and equidominating graphs, Discrete Math. 29 (1980), 47-52, doi:10.1016/0012-365x(90)90286-q.

[55] U. N. Peled and B. Simeone, Polynomial-time algorithms for regular set-covering and threshold synthesis, Discrete Appl. Math. 12 (1985), 57-69, doi:10.1016/0166-218x(85)90040-x. 
[56] U. N. Peled and B. Simeone, An $O(n m)$-time algorithm for computing the dual of a regular Boolean function, Discrete Appl. Math. 49 (1994), 309-323, doi:10.1016/0166-218x(94) 90215-1.

[57] N. Pržulj, D. G. Corneil and E. Köhler, Hereditary dominating pair graphs, Discrete Appl. Math. 134 (2004), 239-261, doi:10.1016/s0166-218x(03)00304-4.

[58] J. Reiterman, V. Rödl, E. Šiňajová and M. Tůma, Threshold hypergraphs, Discrete Math. 54 (1985), 193-200, doi:10.1016/0012-365x(85)90080-9.

[59] D. J. Rose, R. E. Tarjan and G. S. Lueker, Algorithmic aspects of vertex elimination on graphs, SIAM J. Comput. 5 (1976), 266-283, doi:10.1137/0205021.

[60] O. Schaudt, A note on connected dominating sets of distance-hereditary graphs, Discrete Appl. Math. 160 (2012), 1394-1398, doi:10.1016/j.dam.2012.02.003.

[61] O. Schaudt, On graphs for which the connected domination number is at most the total domination number, Discrete Appl. Math. 160 (2012), 1281-1284, doi:10.1016/j.dam.2011.12.025.

[62] O. Schaudt and R. Schrader, The complexity of connected dominating sets and total dominating sets with specified induced subgraphs, Inform. Process. Lett. 112 (2012), 953-957, doi:10. 1016/j.ipl.2012.09.002.

[63] H. Shen and W. Liang, Efficient enumeration of all minimal separators in a graph, Theoret. Comput. Sci. 180 (1997), 169-180, doi:10.1016/s0304-3975(97)83809-1.

[64] Y.-T. Tsai, Y.-L. Lin and F. R. Hsu, Efficient algorithms for the minimum connected domination on trapezoid graphs, Inform. Sci. 177 (2007), 2405-2417, doi:10.1016/j.ins.2007.02.001.

[65] D. B. West, Introduction to Graph Theory, Prentice Hall, Upper Saddle River, New Jersey, 2nd edition, 2001.

[66] J. Wu and H. Li, A dominating-set-based routing scheme in ad hoc wireless networks, Telecommun. Syst. 18 (2001), 13-36, doi:10.1023/a:1016783217662.

[67] J.-H. Yan, J.-J. Chen and G. J. Chang, Quasi-threshold graphs, Discrete Appl. Math. 69 (1996), 247-255, doi:10.1016/0166-218x(96)00094-7.

[68] H.-G. Yeh and G. J. Chang, Weighted connected domination and Steiner trees in distancehereditary graphs, Discrete Appl. Math. 87 (1998), 245-253, doi:10.1016/s0166-218x(98) 00060-2. 


\section{Appendix A A non-connected-domishold split graph whose cutset hypergraph is 2 -asummable}

Based on an example due to Gabelman [32], Crama and Hammer proposed in the proof of [22, Theorem 9.15] an example of a 9-variable 2-asummable positive Boolean function $f$ that is not threshold. From this function we can derive a split graph $G=(V, E)$ on 71 vertices, as follows. Let $V=K \cup I$ where $K=\left\{v_{1}, \ldots, v_{9}\right\}$ is a clique and $I=V(G)-K$ is an independent set. To define the edges between $K$ and $I$, we first associate a nonnegative integer weight to each vertex, as follows: $w\left(v_{1}\right)=14, w\left(v_{2}\right)=18, w\left(v_{3}\right)=24$, $w\left(v_{4}\right)=26, w\left(v_{5}\right)=27, w\left(v_{6}\right)=30, w\left(v_{7}\right)=31, w\left(v_{8}\right)=36, w\left(v_{9}\right)=37$, and $w(v)=0$ for all $v \in I$. Let $\mathcal{S}$ be the set of all subsets $S$ of $K$ such that $w(S) \geq 82$ and let $S_{1}=\left\{v_{1}, v_{6}, v_{9}\right\}, S_{2}=\left\{v_{2}, v_{5}, v_{8}\right\}$, and $S_{3}=\left\{v_{3}, v_{4}, v_{7}\right\}$. (Note that $w\left(S_{i}\right)=81$ for all $i \in[3]$.) Let $\mathcal{H}$ be the hypergraph with vertex set $K$ and hyperedge set given by the inclusion-wise minimal sets in $\mathcal{S} \cup\left\{S_{1}, S_{2}, S_{3}\right\}$. It can be verified that $\mathcal{H}$ has precisely 62 hyperedges (including $S_{1}, S_{2}$, and $\left.S_{3}\right) .{ }^{4}$ The edges of $G$ between vertices of $I$ and $K$ are defined so that set of the neighborhoods of the 62 vertices of $I$ is exactly the set of hyperedges of $\mathcal{H}$.

To show that $G$ is not CD, it suffices, by Proposition 3.4, to show that the cutset hypergraph is not threshold. In the proof of Theorem 9.15 in [22] it is shown that the function $f$ is not threshold, by showing that $f$ is 3 -summable. This corresponds to the fact that the cutset hypergraph of $G$ is 3 -summable, as can be observed by noticing that condition (2.1) is satisfied for $k=r=3$ and for the sets $A_{i}=S_{i}$ for all $i \in[3]$ and $B_{1}=\left\{v_{1}, v_{7}, v_{8}\right\}, B_{2}=\left\{v_{2}, v_{4}, v_{9}\right\}$, and $B_{3}=\left\{v_{3}, v_{5}, v_{6}\right\}$. On the other hand, the fact that $f$ is 2 -asummable implies that the cutset hypergraph of $G$ is 2 -asummable.

\footnotetext{
${ }^{4}$ The following is the list of sets (omitting commas and brackets) of indices of the elements of the 62 inclusionwise minimal hyperedges of $\mathcal{H}: 169,179,189,258,259,268,269,278,279,289,347,348,349,357,358,359$, $367,368,369,378,379,389,456,457,458,459,467,468,469,478,479,489,567,568,569,578,579,589$, $678,679,689,789,1234,1235,1236,1237,1238,1239,1245,1246,1247,1248,1249,1256,1257,1267,1345$, $1346,1356,2345,2346,2356$.
} 